CERN-EP/87-111

24 June 1987

\title{
PERFORMANCE OF THE URANIUM/PLASTIC SCINTILLATOR CALORIMETER FOR THE HELIOS EXPERIMENT AT CERN
}

\author{
T. Åkesson ${ }^{2)}$, A.L.S. Angelis ${ }^{5)}$, F. Corriveau', ${ }^{6)}$ R. Devenish ${ }^{7)}$, G. di Tore ${ }^{2)}$, C.W. Fabjan ${ }^{2)}$, \\ F. Lamarche ${ }^{6}$, C. Leroy ${ }^{6}$, M.L. McCubbin ${ }^{5)}$, N.A. McCubbin ${ }^{8)}$, L.H. Olsen ${ }^{1)}$, M. Seman ${ }^{4)}$, \\ Y. Sirois ${ }^{6}$, R. Wigmans ${ }^{3)}$ and W.J. Willis ${ }^{2)}$
}

\begin{abstract}
We describe the calorimeter system serving the HELIOS experiment at CERN, its calibration, and its performances measured in test experiments. The calorimeter signal for hadrons was found to be proportional to the energy to within $2 \%$ over the energy range $8 \mathrm{GeV}$ to $200 \mathrm{GeV}$. Over the same energy range the energy resolution $\sigma / \mathrm{E}$ scales as $1 / \sqrt{\mathrm{E}}$ for both electromagnetic and hadronic showers. The values found were $(0.215 \pm 0.007) / \sqrt{\mathrm{E}}$ and $(0.337 \pm 0.012) / \sqrt{\mathrm{E}}$, respectively, for the detection of single particles. The signal ratios e/mip and e/ $\pi$ are $0.70 \pm 0.05$ and $0.984 \pm 0.006$, where the latter ratio was found to be dependent on the signal integration time, the quoted number being obtained for a $130 \mathrm{~ns}$ gate. The energy resolution for multiparticle detection was found to be about twice as large as for the detection of single particles in the actual calorimeter configuration. The energy flow logic, designed to provide trigger information on physics quantities such as transverse energy and missing energy, was shown to be very accurate and reliable.
\end{abstract}

(Submitted to Nuclear Instruments and Methods in Physics Research)

1) Brookhaven National Laboratory, New York, USA.

2) CERN, Geneva, Switzerland.

3) CERN, Geneva, Switzerland, and NIKHEF-H, Amsterdam, The Netherlands.

4) CERN, Geneva, Switzerland, and the Slovak Academy of Sciences, Kosice, Czechoslovakia.

5) University College, London, UK.

6) McGill University, Montreal, Canada.

7) On leave from the Nuclear Physics Laboratory, Oxford, UK.

8) Rutherford Appleton Laboratory, Didcot, UK. 


\section{INTRODUCTION}

Large hadron calorimeters play an increasingly important role in the study of particle interactions at very high energies. As the energy of the particles used to study the fundamental structure of matter has increased, the emphasis in the physics analysis has shifted steadily from the classical magnetic momentum analysis of each individual reaction product to more global aspects of the recorded events (jet production, energy flow, missing energy, etc.). Owing to the technological evolution of the last decade, hadron calorimeters can nowadays be designed to meet the extraordinary requirements for these experiments, in terms of trigger selectivity, rate capacity, spatial and energy resolution, etc. Moreover, their performance improves with increasing energy, and their size stays within reasonable limits, even at the highest energies envisaged for the foreseeable future.

In the HELIOS experiment at the CERN Super Proton Synchrotron (SPS), calorimetry plays a key role. The experimental program is twofold: a) a study of lepton production in proton-proton and proton-nucleus reactions, and b) a comparative study of proton-nucleus and nucleus-nucleus reactions at ultra-relativistic energies. The calorimeters cover the full solid angle in the centre-of-mass system, and have the following tasks:

i) To provide an accurate determination of the total transverse energy carried by the particles produced in the interactions over the full solid angle. High-transverse-energy events can thus be selected on line and triggered upon.

ii) To provide an accurate determination of the total energy of the events. This is a crucial ingredient for the recognition of neutrinos produced in the interactions.

iii) To identify isolated individual particles, making use of the characteristic differences in the calorimeter response to electrons, hadrons, and muons.

In addition, the forward part of the calorimeter serves as a shield for the muon spectrometer. The detectors have to operate in a high-rate $(\sim 1 \mathrm{MHz})$ environment.

This paper describes the performance of the HELIOS calorimeter system as observed from the operation in various test beams. Section 2 gives a description of this system. Since the basic design of the calorimeter modules has been given elsewhere [1], we concentrate on the detector layout and on the modifications made to meet the HELIOS requirements. The calibration of the detector is the topic of section 3. In section 4 experimental results are presented on the following subjects: energy resolution for the detection of hadrons and electrons from test beams, signal linearity, rate dependence, leakage effects, energy resolution for the detection of particles produced in a target, tests on the generation of fast signals yielding the total energy, total transverse energy, etc. Conclusions and a summary are presented in section 5 .

\section{THE HELIOS CALORIMETER SYSTEM}

\subsection{Layout}

Figure 1 shows a schematic overview of the calorimeters installed in the HELIOS detector. The target is surrounded by an almost hermetic 'box' of calorimeter modules that cover opening angles up to $96^{\circ}$. The distance from the target to the various walls of the box is approximately $120 \mathrm{~cm}$ in all directions. These walls have a sensitive volume with a depth ranging from 3.2 to 4.2 nuclear interaction lengths. In the forward direction two regions are left open. The first is a $6.3^{\circ}$ conical hole centred around the beam axis. In the interactions most of the available energy will be carried away by particles passing through this hole. This energy is measured in the forward calorimeters which cover an area somewhat larger than the $6.3^{\circ}$ cone, and provide 10.5 nuclear interaction lengths of sensitive depth. The second open region in the otherwise hermetic calorimeter box consists of a slit in the horizontal beam plane, with dimensions $10 \times 85 \mathrm{~cm}^{2}$, corresponding to $0.5 \%$ of the solid angle covered by the calorimeters. The properties of individual particles traversing the slit are measured by 
a separate spectrometer consisting of chambers, magnet, time-of-flight hodoscopes, and finally calorimeter modules.

\subsection{The uranium calorimeter modules}

Four types of calorimeter modules are being used in HELIOS: $\mathrm{Cu} / \mathrm{scintillator}$, $\mathrm{U} / \mathrm{Cu} / \mathrm{scintillator}$, and $\mathrm{U} / \mathrm{scintillator}$ with and without optically decoupled towers. Their position in the detector is indicated in fig. 1. The first two types of module were used before in the Axial Field Spectrometer (AFS), which operated at the CERN Intersecting Storage Rings from 1978 to 1983 . All modules consist of metal plates interleaved with $2.5 \mathrm{~mm}$ thick plastic scintillator plates ${ }^{*}$. The modules containing uranium are divided into two independent sections. The first, the electromagnetic section, is 6.4 radiation lengths deep and contains $2 \mathrm{~mm}$ thick depleted uranium plates. The hadronic section for the U/scintillator modules is 4.0 nuclear interaction lengths $\left(\lambda_{i}\right)$ deep, and the metal plates are depleted uranium ( $3 \mathrm{~mm}$ thick). The hadronic section for the $\mathrm{U} / \mathrm{Cu} /$ scintillator modules is $3.8 \lambda_{\mathrm{i}}$ deep and the $\mathrm{U}$ plates alternate with copper plates $5 \mathrm{~mm}$ thick in the ratio $2: 1$. In the pure $\mathrm{Cu}$ modules the copper plates are $5 \mathrm{~mm}$ thick.

The scintillator plates are read out on two sides by wavelength shifter bars (WLS) made of Plexiglas to which $80 \mathrm{mg} / 1 \mathrm{BBQ}^{* *}$ (shifting agent) is added. These convert the ultraviolet scintillation light to green light. The $\mathrm{Cu}$ modules are read out by two WLS plates. In the other modules the scintillator plates $\left(\sim 120 \times 20 \mathrm{~cm}^{2}\right)$ are read out by six independent WLS plates on both sides, producing a $20 \times 20 \mathrm{~cm}^{2}$ tower structure. The electromagnetic and hadronic sections are read out separately, giving 24 channels per module (see fig. 2). In total, the calorimeter system has 2860 electronic channels. More details about the calorimeter modules are given elsewhere [1].

Before we started to assemble the HELIOS calorimeter with the AFS modules, several of them were examined in test beams. We found that overall performance had deteriorated considerably in the four years since their construction. This phenomenon was due to severe degradation of the optical properties of the scintillator plates. We discovered that the scintillation light continuously produced by the ${ }^{238} \mathrm{U}$ radioactivity, in combination with oxygen which diffuses into the scintillator plates, is responsible for the ageing [2]. The ultraviolet light breaks up the molecular oxygen into its atomic constituents, which are chemically very aggressive. Since the diffusion of oxygen is a slow limiting process, the damage is mainly determined by the duration of exposure and, above a certain lower limit, is independent of the radioactive dose rate. In long-term exposures to a source the decisive influence of the oxygen was clearly demonstrated. In order to avoid further deterioration of the optical quality, a slight overpressure of nitrogen was maintained in each individual module. After une year of operation in this way, it has been checked that there has been no further deterioration. The optical properties of some reference scintillator plates were remeasured and found unchanged. Moreover, the high voltage on the photomultipliers (PMs), as determined from the calibration system (see section 3), has remained constant over the past year, while in the 4 years preceding this modification an increase of 150-200 V was needed to cope with the decreasing light yield.

In total, 13 uranium modules were rebuilt for HELIOS. They were installed in those positions where energy resolution and granularity were most important for the experimental goals. Monte Carlo simulations showed that these positions were the central part of the forward calorimeter and the region around the conical hole. Apart from replacing the yellow scintillator plates by new ones, we also replaced the copper absorber plates in the hadronic section of the module by $3 \mathrm{~mm}$ thick uranium plates. This increased the absorptive power of the sensitive volume per module from 4.06 to 4.24 nuclear interaction lengths, and improved the energy resolution. Futhermore, the reflectors at

*) Altustipe, an acrylic material manufactured by Altulor, Paris, France.

**) Benzimidazo-benzisoquinoline-7-one. 
the short sides of the scintillator plates, which are not read out by WLS plates, were improved compared to the original design. Good light reflection against these edges is essential for ensuring equal signal per $\mathrm{GeV}$ values in all the towers of one module.

In the original design, light produced at a certain point inside a scintillator plate is, in principle, observed by all 12 WLS plates connected to it, and therefore leads to a signal in all 12 PMs viewing the calorimeter section concerned. In order to improve granularity in the most forward modules, the towers were optically decoupled. The $20 \times 120 \mathrm{~cm}^{2}$ scintillator plates were divided into 6 equal parts of $20 \times 20 \mathrm{~cm}^{2}$ by cutting 5 slits of $0.5 \mathrm{~mm}$ width and $18 \mathrm{~cm}$ length, leaving $1 \mathrm{~cm}$ at each side in order to maintain a sufficient mechanical stability for the plate as a whole (fig. 3). We used a $600 \mathrm{~W} \mathrm{CO}_{2}$ laser for this purpose. The plates were mounted on a support that could move very smoothly and accurately in a plane perpendicular to the laser beam. In this way, polished surfaces of a very high quality could be obtained inside the slit ${ }^{*}$. In order to avoid cross talk by light refraction across the slits, reflectors of aluminized Mylar were mounted inside the slits.

The effects of this modification were measured with a set-up shown schematically in fig. 4 . Electrons from $\beta$-decay of ${ }^{106} \mathrm{Ru}$ were used to create scintillation light in a well-defined area of $1 \times 1 \mathrm{~cm}^{2}$ of the scintillator plate. As in the calorimeter, this plate is coupled by WLS bars to the PMs. The $\beta$-electrons are selected by two small scintillation counters that sandwich the scintillator plate. A coincidence between the small counters triggers the reading of the PM signals. The source-trigger counter system can be moved in the $\mathrm{x}$ and $\mathrm{y}$ directions to measure the optical properties of the scintillator plate. Stepping motors perform these movements in a remote-controlled way. In order to make sure that only light created in the scintillator plate and travelling by internal reflection to the WLS bars is detected, the rest of the WLS is covered with black foil.

Figure 5 shows the signals detected by a WLS plate if the light source is moved in the y direction at a fixed distance $\mathrm{x}(10 \mathrm{~cm})$ from the WLS plane. Results are given for the standard plates and for the modified ones. The reflecting slits produce the desired effect: the light is almost completely confined to the tower in which it is produced. The reflectivity of the slit is apparently very good since the total signal changes little when the light source approaches the slit. The signal in the PMs corresponding to the tower in which the light is produced is considerably larger in the decoupled tower design, by $35 \%-100 \%$ depending on where the light is produced. In standard modules, all twelve PMs will, in principle, respond to light produced anywhere in each plate. Decoupling the towers thus improves the signal-to-noise ratio.

The decoupling is, of course, not complete, since light can still pass via the parts that were left uncut in the tower edges (cf. fig. 3). Figure 6 shows the cross-talk pattern, obtained by scanning the whole tower surface with the source-trigger counter system, and recording the signals in the tower where the light is produced as well as in a neighbouring one. Averaged over the tower surface, only a few per cent of the total signal is recorded in the neighbouring towers. In this pattern, one observes the effect of the index of refraction for light going from scintillator to air. The preferred angle $\theta_{1}$ for directly detected light is clearly smaller than the angle $\theta_{2}$ for reflected light.

Three modules were equipped with scintillator plates having the laser-cut slits. They were installed in the centre of the first layer of the forward calorimeter modules, where fine granularity is most needed.

\subsection{The magnetized calorimeter}

The forward region of the 'box' calorimeter is occupied by a calorimetrized dipole magnet (MAGCAL) shown in fig. 7. It consists of an iron yoke of cross-section $64 \times 64 \mathrm{~cm}^{2}$ and $85 \mathrm{~cm}$ in

\footnotetext{
*) We are very much indebted to the Frascati Laboratory in Rome, Italy, for putting their facilities at our disposal, and in particular to Dr. P. Giromini who provided the necessary help.
} 
depth, into which is bored the $6.3^{\circ}$ conical hole already mentioned. The iron yoke is divided into $15 \mathrm{~mm}$ plates alternating with $5 \mathrm{~mm}$ layers of scintillator. Each scintillator layer is divided into 24 azimuthal 'petals', covering approximately $15^{\circ}$ each. In each scintillator layer 4 petals are made of iron to allow the yoke to be bolted together. The azimuthal position of these 4 iron petals is changed in successive layers. The 24 azimuthal towers are read out by WLS bars which are coupled to PMs by light-guides made of $1 \mathrm{~mm}$ diameter optical fibres about $1 \mathrm{~m}$ in length. Optical fibres were used because the path from the end of the WLS bar to the PMs is severely constrained by other detectors, and some degree of flexibility in the light-guide was essential. The scintillator used is Kyowaglas SCSN38 and the WLS bar is Kyowaglas Y7. The yoke has a depth of 3.2 interaction lengths, but many particles traverse a much smaller depth of the yoke, and the geometry of the detector is such that very few hadronic showers are fully contained in the iron. The leakage of these showers is, however, caught by the other calorimeters. The response of a tower to an $8 \mathrm{GeV}$ electron beam shows an energy resolution $(\sigma / \mathrm{E})$ corresponding to $0.25 / \sqrt{\mathrm{E}}$, which is as expected for our sampling thickness.

\subsection{Signal handling}

The anode signals of the PM tubes are used for two purposes. Two thirds of the signal is sent to an analogue-to-digital converter (ADC) which records the total integrated charge. This is the signal which is used for the off-line analysis. The remaining one third is diverted into the Energy Flow Logic (EFL) and is used on line for triggering purposes.

The goal of the EFL is to provide a trigger on the total transverse energy, the total missing energy, and the total missing transverse momentum of an event. This is accomplished by fast analogue summing of the physical quantities $E_{t o t}, E_{T}$, and $P_{T}^{2}$, obtained from the PM signals of the various calorimeters in the experiment. The final analogue sums are fed to digital comparators via flash ADCs (FADCs) for use in the parallel-trigger processor. In order to obtain, at the trigger level, physical quantities that will approximate as accurately as possible their true values, the raw energy signals have been weighted with Monte Carlo generated weights which take into account the geometrical position of the energy deposition as well as the effects introduced by the finite spatial and energy resolutions of the calorimeters, and by light attenuation in the scintillator.

The individual physical quantities are formed as follows: for the $E_{\text {tot }}$ quantity, the pulse height from each element of the calorimeter is added with equal weights, and the gain of the summing chain is adjusted according to whether the signal is digitized in a 7-bit or 9-bit FADC. For the $P_{T}^{2}$ quantity four sums, labelled $\mathrm{P}_{\mathrm{x}}^{+}, \mathrm{P}_{\mathrm{x}}^{-}, \mathrm{P}_{\mathrm{y}}^{+}, \mathrm{P}_{\mathrm{y}}^{-}$, are formed. Each element of the calorimeter contributes to only two of these sums, depending on its azimuthal coordinates, with a weight relative to $E_{\text {tot }}$ proportional to $\sin \theta_{\mathrm{i}} \times \sin \phi_{\mathrm{i}}$ or $\sin \theta_{\mathrm{i}} \times \cos \phi_{\mathrm{i}}$ for the y or $\mathrm{x}$ components, respectively (see fig. 1). Here $\theta_{\mathrm{i}}$ and $\phi_{\mathrm{i}}$ are the effective polar and azimuthal angles of the element $i$. Apart from this geometrical factor, the weights are adjusted to account for the effects mentioned before. Each of the four signals is digitized independently and digital arithmetic is used to form the quantity

$$
\mathrm{P}_{\mathrm{T}}^{2}=\left(\mathrm{P}_{\mathrm{x}}^{+}-\mathrm{P}_{\mathrm{x}}^{-}\right)^{2}+\left(\mathrm{P}_{\mathrm{y}}^{+}-\mathrm{P}_{\mathrm{y}}^{-}\right)^{2}
$$

For the $\mathrm{E}_{\mathrm{T}}$ quantity, the pulse height from each element is added with a weight relative to $\mathrm{E}_{\text {tot }}$, of which the geometrical part is proportional to $\sin \theta_{\mathrm{i}}$. In order to trigger on total missing energy, the $E_{\text {tot }}$ comparator is inverted so as to trigger on the difference between the total energy and the beam energy.

The actual analogue summing is carried out in three or four stages depending on the calorimeter component in question, and the corresponding electronic summing units are termed $\Sigma_{1}, \Sigma_{2}, \Sigma_{3}$, and $\Sigma_{4}$. The weights provided by the Monte Carlo were used to calculate resistor values for each 
individual input to the first stage of summing ( $\Sigma_{1}$ level). To achieve the desired accuracy in the trigger signals, high-precision resistors (differing by less than $1 \%$ from their nominal values) were used for the splitting of the primary current and for the weighting factors. The splitting of the PM signal and the input stage to the first level summing are shown schematically in fig. 8a. Apart from the four weighting resistors for the four quantities $E_{t o t}, P_{x}, P_{y}$, and $E_{T}$, calculated for each element of the calorimeter, a fifth resistor $\mathrm{R}_{5}$ is also used to match the input impedance of the first level summing to $50 \Omega$.

A schematic representation of the cabling and summing layout for part of the calorimeter is shown in fig. $8 \mathrm{~b}$. The other components have a very similar structure. There are four $\Sigma_{1}$ units per quadrant where energy coming from the hadronic part of a module is summed (e.g. 1H11), and four where energy coming from the electromagnetic part of a module is summed (e.g. 1E11). The outputs of up to four $\Sigma_{1}$ units are fed into the second level summing $\left(\Sigma_{2}\right)$, and there is one $\Sigma_{2}$ unit per quadrant for hadronic energy summing and one for electromagnetic energy summing. The outputs of up to four $\Sigma_{2}$ units are fed into the third level summing $\left(\Sigma_{3}\right)$, where the separate sums of hadronic and electromagnetic energies are obtained. There are two $\Sigma_{3}$ units per component. The final summing of hadronic plus electromagnetic energy is made in one $\Sigma_{4}$ unit per calorimeter component. The output of the $\Sigma_{4}$ is sent, via a differential driver unit, to $150 \mathrm{~ns}$ shaping amplifiers in the counting room, and from there, via adapter units which provide for fine adjustment of the summing chain gain, into the FADCs and the digital part of the EFL. The gains of the summing units corresponding to a given calorimeter component are optimized to keep the electronic noise at a minimum and at the same time use the full FADC range for the signals expected from that component. The overall energy scale is fixed by the calibration of the charge ADCs.

The logical connection of the EFL digital units is shown in fig. 8c. The signals for each physical quantity and from each one of the calorimeters are fed into FADCs. They are then added together using digital ADDER modules to provide values for the physical quantities $\mathrm{E}_{\text {tot }}, \mathrm{P}_{\mathrm{x}}^{+}, \mathrm{P}_{\mathrm{x}}^{-}, \mathrm{P}_{\mathrm{y}}^{+}$, $\mathrm{P}_{\mathrm{y}}^{-}$, and $\mathrm{E}_{\mathrm{T}}$. Digital SUBTRACTOR modules and memory look-up units are used to provide a value for the physical quantity $\mathrm{P}_{\mathrm{T}}^{2}$. These values are then compared with a set of predetermined thresholds in order to decide whether a trigger condition is satisfied or not. The digital EFL units are under CAMAC control which allows, via selection of ADDER inputs and via the use of all five available thresholds on each COMPARATOR module, a great variety of EFL triggers to be defined. Both 7-bit and 9-bit FADCs are used, in order to cope better with the dynamic range of the energy sum signals.

\section{THE ENERGY CALIBRATION OF THE CALORIMETER}

High-precision calorimetry requires a major effort concerning the energy calibration of the system. Problems associated with the calibration are generally non-trivial to resolve. Two aspects are important to obtain a satisfactory result:

i) tuning the PMs such that the energy deposition of a given particle always produces the same signal (measured in picocoulombs) independent of the calorimeter module in which it is detected, and

ii) setting the absolute energy scale.

Among the problems which arise in practice, we mention:

i) instability of the PMs, which require frequent checking and adjustment;

ii) the scintillation light is attenuated both in the scintillator plates and in the WLS; therefore, the calorimeter response also depends on the impact point of the particle, the angle of incidence and, for hadron showers, the depth at which the shower starts developing; 
iii) the absolute signal-to-energy conversion is in general quite different for muons, electrons, photons, and hadrons (for the latter class of particles, moreover, the signal in hadron calorimeters is in general not proportional to the particle energy).

The use of uranium absorber plates offers a major advantage compared to other materials, since the radioactivity provides a convenient, stable, homogeneously distributed source of signals that can be used for a relative calibration of the complete optical chain.

We have developed the following calibration procedure:

i) First, each individual charge ADC channel is calibrated using a precision pulse generator. The charge equivalent per ADC count is thus accurately determined for each individual channel.

ii) Next, measurements of the radioactive decay of uranium nuclei (the 'uranium noise') are performed for all PMs. In order to get signals that are sufficiently accurate with respect to the $\mathrm{ADC}$ pedestals, a relatively long gate time of $10 \mu \mathrm{s}$ is used instead of the $0.13 \mu \mathrm{s}$ which is the standard value for shower detection. The gains of the PMs, i.e. the ratios $\mathrm{MeV} / \mathrm{pC}$, are equalized for PMs that read out towers of the same structure by using the mean $\mathrm{ADC}$ values of the uranium noise signal distributions. By towers of the same structure we mean, for example, all electromagnetic towers, or all hadronic towers of the $\mathrm{U} / \mathrm{Cu} / \mathrm{scintillator}$ modules, or all hadronic towers of the U/scintillator modules. By using the uranium noise only, one can equalize the gains, but one cannot determine the absolute value of the ratios $\mathrm{MeV} / \mathrm{pC}$. Since the numbers of scintillator plates, the amount of uranium, and the uranium-plate thicknesses are different for the various categories mentioned before, these gain values will differ as well. In order to determine them one needs calibration runs with particles of a well-defined energy.

iii) The last step in the calibration procedure, therefore, consists of a determination of the absolute values of the ratios $\mathrm{MeV} / \mathrm{pC}$ for the various types of modules with beams of different energies. Since the electromagnetic sections are thin $\left(6.4 \mathrm{X}_{0}\right)$, charged particles will, in practice, always produce a signal in both the electromagnetic and hadronic sections of a module, and therefore one can only calibrate both sections simultaneously.

In order to obtain the absolute values of the calibration constants, we used tagged electron beams from the CERN SPS, at energies of $8,17,24,32$, and $45 \mathrm{GeV}$, and cosmic muons. Electrons have the advantage that their total energy is deposited in a limited region fully contained by the module, with comparable fractions in the two sections.

The calibration constants A and B for the electromagnetic and hadronic sections were calculated by minimizing the width of the total signal distribution, i.e. by minimizing the quantity

$$
Q=\sum_{j=1}^{N}\left[E(\text { beam })-A \sum_{i=1}^{n} S_{i j}{ }^{\text {em }}-B \sum_{i=1}^{n} S_{i j}{ }^{\text {had }}\right]^{2},
$$

where $\Sigma S^{e m}$ and $\Sigma S^{\text {had }}$ are the sums of all the ADC counts in the towers $i$ of the electromagnetic and hadronic sections that contribute to the measured signal for event $j$.

The method yielded rather large errors on the values of $\mathrm{A}(30 \%)$ and $\mathrm{B}(15 \%)$, but their strong negative correlation ( $-92 \%$ ) meant a $4 \%$ uncertainty only in the absolute reconstructed event energy owing to the large event-by-event fluctuations in the energy sharing between both sections. Mean energies, however, as will be shown in section 4.1 .2 , are reconstructed to better than $2 \%$.

The procedure was repeated at each individual beam energy. The following essential points illustrate some difficulties one faces when aiming at high-resolution calorimetry:

i) A small but significant difference between the calibration constants for different types of stacks was found.

ii) The values of $\mathrm{A}$ and $\mathrm{B}$ found from electrons were slightly energy dependent.

iii) The calibration constants found from electrons were inconsistent with those from muons. 
The first effect is probably the least disturbing one. There are many possible explanations. The electrons deposit their energy in a limited region of the calorimeter volume and therefore the signal relative to the uranium noise is rather sensitive to anomalies in, for example, the thickness of the active or passive layers. The difference that we observed between the $\mathrm{A}$ and $\mathrm{B}$ values of the module with the optically decoupled towers and the uranium module with the unmodified scintillator plates is also partly due to the fact that the reflection of the aluminized Mylar foils in the slits (cf. section 2.2) is not perfect. The effect of this on the locally produced light from showers differs from that on the uniformly distributed light generated by uranium decay. The observed overall effect goes in the expected direction, i.e. there are somewhat larger (by 8\%) A and B values for the decoupled towers.

The other effects have a very fundamental meaning. The fact that different $\mathrm{A}$ and $\mathrm{B}$ factors, and also a different B/A ratio, have to be used for different electron energies has the consequence that signal linearity is violated. Signal linearity for electron-shower detection is a fundamental requirement. It looks as if one cannot find calibration constants that fulfil this requirement and optimize the energy resolution for electromagnetic showers at the same time. The ratio B/A went up with the energy by about $20 \%$ over the energy range studied.

Cosmic muons only allow a value of B/A to be derived, since the energy deposited in the modules depends in a complicated way on the energy of the muon (section 4.1.2) which is not known. The $\mathrm{B} / \mathrm{A}$ ratio from muon calibration was found to be $\sim 21 \%$ larger than the (average) value from the electron runs. The questions are: Where do these differences come from? and Which calibration factors should be used?

There are two contributions to these effects, an instrumental one which could eventually be avoided, and a contribution that has to do with the physics of shower development and, therefore, cannot be avoided. The instrumental part of the explanation is caused by the light attenuation in the WLS of the hadronic section. The light production by both muons and uranium noise is uniform as a function of depth. Light from the electron shower tail is produced in the part of the hadronic section furthest from the PM, and is therefore more attenuated than the average for uranium noise and muons. From the measured WLS attenuation characteristics we found that an effect of 10-15\% has to be expected on the B value for electrons. The longitudinal inhomogeneity of the WLS might be resolved by properly filtering the light transmitted from the scintillator into the WLS [3].

The physics part of the explanation was pointed out elsewhere [4]. It turns out that in a sampling calorimeter, the fraction of the electromagnetic shower energy converted into a measurable signal changes with depth. In the case of uranium absorber a very considerable decrease occurs. This is due to the soft $\gamma$ component of the shower, for which the calorimeter response (signal per unit of energy) is much lower than for minimum ionizing particles (mip's). This means that a given amount of energy deposited by the fast (more mip-like) part of the shower in the electromagnetic section will yield a larger signal than the same energy deposited by the soft tail in the hadronic section. The effect of this on our calibration result is energy dependent: if the electron energy is increased, the particles in the fraction of the shower deposited in the hadronic section becomes on an average more energetic, which results in a larger response. Experimentally, we should then find a larger B/A ratio, which is indeed the case. This phenomenon creates a very fundamental problem: the relation between the energy deposited by the shower and the resulting calorimeter signal is different for the two sections of the calorimeter and, moreover, energy dependent. This was clearly confirmed by EGS4 calculations with which we simulated the development of electromagnetic showers in our calorimeter. Averaged over all energies the hadronic/electromagnetic signal ratio is predicted to be $30 \%$ smaller than the ratio of the energies deposited in the two sections.

The combined result of these two contributions explains the experimentally observed discrepancies between the electron and muon calibrations and the energy dependence of $A$ and $B$ in the electron calibration. In practice, we decided to use the $\mathrm{B} / \mathrm{A}$ value from the muon calibration, 
fixing the $A$ and $B$ values such as to reproduce best the electron beam energies over our measured range. This guarantees good proportionality between the total electron signal (expressed in energy units) and the particle energy. Any other choice would only have a limited meaning for electrons of a particular energy, and for nothing else. An inevitable consequence of this choice is that, while the overall linearity is preserved, the apparent energy deposited by electromagnetic showers in separate segments is slightly offset with these calibration constants. This is also valid for hadronic showers, where the calorimeter response analogously changes with shower depth.

We investigated the effect of changing the $B / A$ value on energy resolution and on the $\mathrm{e} / \pi$ signal ratio. Results are given in fig. 9. The dashed line gives our chosen $B / A$ value. Figure 9 a shows what happens to the quantity that we minimized in order to find $\mathrm{B}$ and $\mathrm{A}$ from the electron measurements, i.e. the $\sigma_{\mathrm{rms}}$ of the signal distribution. It shows that for electrons the minimum is found for a lower $\mathrm{B} / \mathrm{A}$ value, and how $(\mathrm{B} / \mathrm{A})\left(\sigma_{\min }\right)$ shifts with energy. Because of the arguments just given, the $\sigma_{\mathrm{rms}}$ found as a minimum value cannot be interpreted as an energy resolution but as the width of the distribution of the total number of ADC counts. Figure $9 \mathrm{a}$ also shows that the hadronic energy resolution is very insensitive to the forward-backward gain balancing. This is because the energy fraction deposited by hadrons in the electromagnetic section $\left(0.2 \lambda_{\mathrm{i}}\right)$ is very small. This also explains what is observed in fig. $9 \mathrm{~b}$, where the effect of the $\mathrm{B} / \mathrm{A}$ value on the $\mathrm{e} / \pi$ signal ratio is shown. If more weight is given to the part of the shower detected in the hadronic section, the $\mathrm{e} / \pi$ signal ratio obviously will decrease.

The lesson to be learned from all this is twofold.

i) Showers should not be used to intercalibrate sections of a longitudinally segmented calorimeter.

ii) In such a calorimeter, calibrated with muons, only the total shower energy recorded is meaningful, while the signals in the individual segments cannot be fully interpreted as energy information.

The shower age-dependent calorimeter response therefore also implies that the weighting factors used in algorithms [5] to improve the energy resolution off line, using the longitudinal shower information, should be energy dependent.

Once the values for $\mathrm{A}$ and $\mathrm{B}$ are fixed, the uranium noise signal can be used to set and maintain the required gain. The high voltages applied to the PMs are chosen such that the gain factors $\mathrm{A}$ and $\mathrm{B}$ are the same for all channels, except in the very forward region where the gains are deliberately set lower to accommodate the much higher energy deposition. The gain stability is checked daily by measuring the uranium noise signal, which is maintained at a fixed number of ADC counts for each specific channel.

The response of the 24 tubes of the MAGCAL is checked and maintained by two systems: light diodes and a radioactive source. One fibre of each light-guide is coupled to a light diode. The light passes down the fibre, along the WLS bar, where it is reflected at the end and returns to the PM. In order to control systematic effects in the light diodes, each diode feeds four channels, and the temperature of the diodes is monitored. In order to check the șcintillator response, a $740 \mathrm{MBq}{ }^{60} \mathrm{Co}$ source is pulled round the front face of the MAGCAL. This source samples the first few layers of scintillator. Experience with the stability of the system so far has been very good.

Maintaining the correct energy response (i.e. equal gains in $\mathrm{MeV} / \mathrm{pC}$ ) is of great importance since part of the PM signal is used to trigger on physics quantities through analogue summing of very many such signals. The absolute energy scale was set by scanning the different types of petals with an $8 \mathrm{GeV}$ electron beam.

\section{RESULTS OF TEST BEAM MEASUREMENTS}

The performance of the calorimeter system was tested with secondary and tertiary negatively charged particle beams from the SPS. Electron beams were available for energies from $8 \mathrm{GeV}$ to 
$70 \mathrm{GeV}$. Above $70 \mathrm{GeV}$ the electron content of the beam was negligible. For most of the experiments the beam was sent directly into the centre of a $20 \times 20 \mathrm{~cm}^{2}$ tower of the central module of the forward calorimeter, which is optically decoupled from the other towers (section 2.2). For some performance tests the beam was focused on a target placed $3.9 \mathrm{~m}$ upstream of the front face of the forward calorimeter (fig. 1), in which interactions were defined with a scintillator hodoscope/silicon pad array arrangement.

\subsection{The calorimeter response to single particles}

The detailed discussion on calorimetry performance based on the results from the modules with decoupled towers are presented in the following subsections.

\subsubsection{Particle identification}

The identification of the type of particle giving the signal in the calorimeter was obtained using the tagging information coming from a Cherenkov differential counter with achromatic ring focusing (CEDAR), capable of separating electrons from pions efficiently up to $45 \mathrm{GeV}$. Secondly, the longitudinal and transverse signal pattern in the calorimeter itself shows very characteristic differences, owing to the decoupled tower structure. A powerful method of distinguishing electrons from pions comes from the longitudinal shower development. The requirement that $>30 \%$ of the total energy be deposited in the electromagnetic section of the first layer of calorimeter modules removes all pions, whilst requiring $<3 \%$ removes basically all electrons. Muons are very easily recognized since they deposit only a few $\mathrm{GeV}$ of energy in the calorimeter.

The transverse difference is illustrated in fig. 10. Figure 10a shows the signal distribution in the calorimeter tower that was hit by a beam of $45 \mathrm{GeV}$ particles, composed of muons, pions, and electrons. The transverse dimensions of the electromagnetic shower are such that practically all the light is produced in this tower $\left(20 \times 20 \mathrm{~cm}^{2}\right)$. The same is true for the muons. Because of the almost complete absence of light sharing, more than $90 \%$ of the signal is recorded by the PMs viewing this tower, for these particles. The transverse dimension of hadron showers, being much larger, results in a considerably smaller fraction of the signal being observed in the hit tower for these particles, as seen in fig. 10a. If the signals of all towers are summed up, the e and $\pi$ peaks overlap, indicating that the $e / \pi$ signal ratio is close to one (fig. 10b). The hadron signal distribution in fig. $10 \mathrm{a}$ is much broader than the one for the total hadron signal, because of the fluctuations in the fraction of energy deposited in the $20 \times 20 \mathrm{~cm}^{2}$ region considered here.

\subsubsection{Energy resolution, $e / h$, and signal linearity}

The energy resolution for electrons and pions was measured with beams of $8,17,24,32,45,70$, and $200 \mathrm{GeV} / \mathrm{c}$. The $8 \mathrm{GeV}$ data contained too few pions for a useful analysis, whereas the $200 \mathrm{GeV}$ data contained too few electrons. The beam intensity was kept at 100 particles per burst $(2.6 \mathrm{~s})$, except at $200 \mathrm{GeV}$ where the intensity could not be reduced below $10^{4}$ particles per burst. Typically, $10^{4}$ events were recorded at each energy. Pions and electrons were separated using the CEDAR information and the longitudinal and lateral shower characteristics. In order to avoid longitudinal leakage effects, we removed events where less than $95 \%$ of the energy was deposited in the first 4.2 nuclear interaction lengths (late shower starting point). The $\sigma$ of the signal distribution was determined from a fit to a Gaussian distribution over the approximate range (mean $-3 \sigma$, mean $+3 \sigma\}$. The results are shown in fig. 11 , where $\sigma / \sqrt{\mathrm{E}}$ is given as a function of energy, for electrons and pions. The error bars include systematic uncertainties. Within experimental uncertainties the value of $\sigma / \sqrt{\mathrm{E}}$ is independent of the particle energy. A least squares fit to the data points gives $\sigma / \mathrm{E}=(0.337 \pm 0.013) / \sqrt{\mathrm{E}}$ for pions and $(0.215 \pm 0.007 / \sqrt{\mathrm{E}}$ for electrons, with $\mathrm{E}$ in $\mathrm{GeV}$. For comparison we also show a set of typical results for $\pi$ detection obtained with an iron/scintillator 
non-compensating calorimeter [5]. Our results show a considerably better hadron energy resolution, even compared to the results obtained by ref. [5] after an off-line weighting procedure intended to correct for the non-Gaussian event-by-event fluctuations in the fraction of energy spent on $\pi^{0}$ production.

Figure 12 shows the e/ $\pi$ signal ratio as a function of energy. The point at $200 \mathrm{GeV}$ was obtained by assuming that the response to electrons continues to be linear with the electron energy beyond $70 \mathrm{GeV}$, the highest energy for which electron beams were available. As before, the error bars include systematic uncertainties. Again, over the energy range studied in these test experiments the result is constant within experimental uncertainties. An average value of $0.984 \pm 0.006$ is found.

The linearity of the calorimeter response with energy was investigated as well. In fig. 13, the signal per unit of energy is given, as a function of energy, for detection of pions in the energy range $17 \mathrm{GeV}$ to $200 \mathrm{GeV}$. There is no indication of a deviation from proportionality larger than $2 \%$ over this interval. The numerical results on energy resolution, signal linearity, and $\mathrm{e} / \pi$ signal ratio are summarized in tables $1 \mathrm{a}$ and $1 \mathrm{~b}$ for both types of $\mathrm{U} / \mathrm{scintillator}$ calorimeter modules, with and without optically decoupled towers, respectively.

Severe non-linearities have been observed in tests of calorimeters with $e / \pi$ ratios very different from one. For comparison, fig. 13 shows results reported by the CDHS Collaboration, obtained from tests of their iron/scintillator calorimeter [5]. These effects, which amount to $20 \%$ over the energy range $10 \mathrm{GeV}$ to $100 \mathrm{GeV}$, are explained by the fact that the average fraction of energy spent on $\pi^{0}$ production in the shower development increases with energy [4]. The weighting algorithm referred to before was apparently not adequate to remove such non-linearities (fig. 13). The best performance of a hadron calorimeter is expected if the average response to the electromagnetic and purely hadronic parts of the hadron showers are equal [6]. We will refer to this condition as $\mathrm{e} / \mathrm{h}^{\mathrm{intr}}=1$. Strictly speaking, for perfect detectors only, this condition is equivalent to the requirement that the average calorimeter responses to electrons and pions be equal $(e / \pi=1)$. Perfect detectors means devices which do not suffer from inhomogeneities in the light collection, which is not the case in practice. The electrons produce light in the very beginning of a calorimeter module, while pions (including the electromagnetic $\pi^{0}$ part of the showers) cause a more uniform illumination in depth. The measured $\mathrm{e} / \pi$ value is, therefore, sensitive to the optical properties of the WLS bars. In order to avoid these problems, one needs pion beams spanning an energy range as large as possible, at least an order of magnitude. Signal non-linearity and deviations from $1 / \sqrt{E}$ scaling of the energy resolution for hadron detection are the experimental probes of the difference between $\mathrm{e} / \pi^{\text {meas }}$ and $e / \pi^{\text {intr }}$ (cf. discussion in ref. [4]). Our results shown in fig. 11 and fig. 13 indicate that $\left|\mathrm{e} / \mathrm{h}^{\text {intr }}-1\right| \leq 0.05$ at a $95 \%$ confidence level.

\subsubsection{The e/mip ratio}

Besides electrons and pions, the beams used to determine the calorimeter performance also contained muons (fig. 10). This made it possible to study the relative calorimeter response to muons and electrons in the same experimental conditions. To first order one would expect the $e / \mu$ signal ratio to be one $e^{*}$, but usually a considerably smaller value is found, the explanation of which has given rise to many speculations in the literature [6].

A minimum ionizing particle loses on an average $901 \mathrm{MeV}$ in the sensitive volume of one of our forward U/scintillator stacks [7]. Having set the absolute energy scale by electrons, it is therefore sufficient to determine the apparent energy deposited by the muons and compare it with $901 \mathrm{MeV}$ (or $1.802 \mathrm{GeV}$ if the signals are summed for the whole forward calorimeter) in order to find the $\mathrm{e} / \mu$

*) The muons in general lose only a small fraction of their energy in the calorimeter. By the $\mathrm{e} / \mu$ ratio we mean the relative calorimeter response to muons and to electrons that carry an energy equal to the one lost by the muons. 
signal ratio. We used the total signal observed in the first $8.5 \lambda_{\mathrm{i}}$ forward calorimeter for this analysis, since a) the background contribution of late hadron showers to the muon signal is eliminated in this way, and b) the muon signal is twice as large as the signal in the first layer of stacks, where all the energy of the electron showers is deposited (figs. 10a and b). Figure 14 gives the muon signal distributions for various energies. It shows two features:

i) the signal distribution becomes less symmetric if the muon energy is increased, and

ii) the average apparent energy increases with the muon energy.

In fig. 15 the average apparent muon energy is given as a function of beam energy. The values should be compared with the $1.802 \mathrm{GeV}$ of an average minimum ionizing particle. Their ratio, therefore, steeply increases with the energy. This observation strongly suggests that a comparison of $\mathrm{e} / \mu$ signal ratios obtained by different experiments is only meaningful if the muon measurements were done at the same, well-defined energies.

The observed energy dependence means, moreover, that high-energy muons cannot be considered as minimum ionizing particles. The energy loss per unit length may be considerably larger than the minimum ionizing value if the muons are extremely relativistic [8]. This is due to phenomena such as $\delta$-ray emission (relativistic rise), bremsstrahlung, pair production and, at the very highest energies, nuclear reactions. The contribution of such effects is very energy and material dependent (fig. 16). The evaluation of the consequences for the muon signal in a detector with very high $Z$ absorber material $\left({ }^{238} \mathrm{U}\right.$ ), and low $\mathrm{Z}$ readout (plastic scintillator) is therefore not trivial. We estimated the increase of the calorimeter response relative to the minimum ionizing value as follows. As far as the direct ionization (including $\delta$-ray emission) is concerned, we used the $\mathrm{dE} / \mathrm{dx}$ values for PMMA ${ }^{*}$, assuming that the range of the much more abundantly produced $\delta$-rays in uranium is sufficiently short to be considered a local energy deposit which has no effect on the calorimeter signal. Our $\mu / \mathrm{e}$ signal ratio was determined as the ratio of the measured muon signal (fig. 15) to a minimum ionizing particle signal. When removing the relativistic rise effect, we see a decrease of the effective $\mu / \mathrm{e}$ ratio (cf. the open circles in fig. 17). In addition, the $\mu / \mathrm{e}$ ratio becomes much less energy dependent. For the other effects which play a role we assumed that what is happening in the absorber plates will affect the signal as well. The extra energy deposited by bremsstrahlung, pair production, and nuclear reactions was assumed to be sampled. This correction mainly affects the highest energy points. The fully corrected $\mu$ /e signal ratios calculated in this way (the crosses in fig. 17), may now be considered as real mip/e signal ratios. They are no longer significantly dependent on the energy. The e/mip value extracted from a least squares fit to the data points is $0.70 \pm 0.05$, the error including an estimate of the systematic uncertainties.

\subsubsection{The rate dependence}

The results mentioned in the previous subsections were obtained at very low rates of incident beam particles ( $10^{1}$ to $10^{4}$ per second). At high incident rates special precautions have to be taken to avoid a serious degradation of the calorimeter performance. Rate effects were systematically studied with the $200 \mathrm{GeV} \pi^{-}$beam. The particles were sent to the centre of the middle $20 \times 20 \mathrm{~cm}^{2}$ tower of the forward calorimeter. The average signal for this tower increased by $18 \%$ as the beam intensity was increased from $3 \times 10^{3}$ to $2 \times 10^{5}$ particles per second. For a neighbouring tower, and for one located $80 \mathrm{~cm}$ away in the lateral direction, the signals did not change significantly with the beam intensity. As described in detail below, this rate dependence in the middle tower signal is mainly due to a gain shift in the PMs (which we were able to overcome) and partly to unavoidable pile-up effects. This pile-up effect is due to overlap in time of the light produced by different particles. This may happen if two particles hit the detector sufficiently close in time, and also if induced radioactivity (e.g. fission products) contributes at a significant level to the light yield. We protected ourselves

*) Polymethyl methacrylate. 
against overlapping events by requiring no other triggered event within a $\pm 1 \mu \mathrm{s}$ window. In order to see whether this protection was sufficient we studied the calorimeter signals as a function of the charge ADC gate delay and width. The results are given in fig. 18 for electrons and pions. One sees that the light production in a shower extends significantly beyond $130 \mathrm{~ns}$, both in electromagnetic and hadronic showers (fig. 18a), although the fraction of light produced after $130 \mathrm{~ns}$ is slightly larger in the latter case (fig. 18b). Two phenomena play a role. Firstly, some fraction of the total energy deposited in a hadronic shower comes from captured thermalized neutrons. Thermalization of neutrons in a uranium calorimeter is a process with a $1 \mu \mathrm{s}$ time scale [9]. This process does not play a role in electromagnetic showers, and therefore probably explains the small differences in the time structure of the electromagnetic and hadronic showers, which have also been observed in other experiments [3]. The remainder of the pile-up effect is caused by the optical part of our signal formation, and in particular by the wavelength shifting mechanism which has a slow component (fluorescence). Figure 18b shows that about $5 \%$ of the total light production occurs beyond $1 \mu \mathrm{s}$ after the particle entered the detector, so that for high rates $(\geq 0.1 \mathrm{MHz})$ this effect is expected to make a contribution.

Pile-up will cause a base-line shift in our energy scale. We checked this by recording data triggered by a randomly pulsed system, rejecting of course triggers generated within the $2 \mu$ s interval defined by real events. The energy equivalent of the ADC readings for these 'empty' events showed a large increase as the $200 \mathrm{GeV} \pi^{-}$beam intensity was increased from $3 \times 10^{3}$ to $2 \times 10^{5}$ particles per second. However, this pile-up effect explains only $3 \%$ of the total $18 \%$ shift observed in the middle tower.

The main contribution to the rate dependence was due to the PMs themselves. Our PMs were designed to operate at a maximum average current of $200 \mu \mathrm{A}$. For the PMs of the middle tower this current (200 photoelectrons per $\mathrm{GeV}$ at a gain of $10^{6}$ ) corresponds to the current delivered in detecting $200 \mathrm{GeV}$ particles at a rate of $80 \mathrm{kHz}$. At higher rates, where the PMs are operating beyond their design value, gain shifts may therefore be expected. Such effects were clearly observed and appeared to be time dependent on a scale of a fraction of a second. It is interesting to note that at very high light intensities and currents the gain of the PM, and hence the apparent energy, increase significantly, in contrast to the normal 'sagging' effect. For high intensities the signal rises and reaches a plateau value after about $0.5 \mathrm{~s}$. The gain shift observed amounts to about $20 \%$ for the highest intensities studied, where the current delivered by the PM is an order of magnitude above the design value. Given this very reproducible behaviour, it is understandable that the energy resolution improved considerably if the events taken in the first $0.5 \mathrm{~s}$ of the burst were discarded or corrected ior in the analysis (fig. 19).

Since the HELIOS physics program requires beam intensities and energies which vary widely, we have adopted a simple and flexible solution to this problem by varying the high voltage and inserting grey filters to reduce the incident light. In a recent exposure with ${ }^{16} \mathrm{O}$ ions at $3.2 \mathrm{TeV}$, the central tower of the forward calorimeter was equipped with grey filters which reduce the amount of light reaching the PM by a factor of 15 . This allowed the PMs to be operated in their linear regime and yet not saturate when detecting the light produced at an energy deposition of up to $2 \mathrm{TeV}$ in the middle tower. Gain shifts at high intensities (up to $0.1 \mathrm{MHz}$ ) were not observed, and the total energy resolution for detecting the products of interacting ${ }^{16} \mathrm{O}$ ions was $1.9 \%$ at these intensities (fig. 20). Using the energy calibration obtained at low energies ( $8-45 \mathrm{GeV})$, we found the centre of the energy distribution of the oxygen ions to be at $3.15 \mathrm{TeV}$, which means that the signal linearity is accurately maintained over three orders of magnitude of hadronic energy. 


\subsubsection{Leakage effects}

In the study of leakage in hadron showering, we distinguish between two different cases:

i) leakage out of the back of the calorimeter due to incomplete shower containment which will arise if the primary hadron penetrates very deeply before undergoing its first nuclear reaction, and

ii) leakage through the front face of the calorimeter, or albedo, an effect that is presumed to be mainly due to soft neutrons abundantly produced during the development of the shower [10].

The first case was studied by measuring the fraction of the energy remaining after the first row of forward U/scintillator ( $4.3 \lambda_{\mathrm{i}}$ thick) calorimetry. Figure 21 shows this energy spectrum for 24,45 , 70 , and $200 \mathrm{GeV}$ incoming pions.

\subsection{Multiparticle detection}

In practice, calorimeter systems are used to detect particles produced in interactions, which may enter the detector at all possible angles and impact points. Moreover, several of them may develop showers that are so close in space that separating them is impossible, as for example in jets. The tests described so far ignore these complications. The calorimeter performance can be expected to deteriorate with respect to the 'ideal' case of single particles entering the detector at a fixed point and incident angle. This is due to increased contributions of lateral inhomogeneities in the light collection caused by light attenuation in the scintillator and also due to the 'dead' space occupied by wavelength shifters and module frames ( $8 \%$ of the active volume). The problem in determining the performance under realistic experimental conditions is that determination of figures of merit, such as energy resolution and signal linearity, is in principle not possible since one cannot calibrate with respect to a particle composition which is known beforehand. We have studied these effects by measuring the particles produced in interactions caused by $200 \mathrm{GeV} / \mathrm{c}$ negative pions in a thin $\left(0.02 \lambda_{\mathrm{i}}\right)$ tungsten plate installed at the nominal HELIOS target position, which is about $3.9 \mathrm{~m}$ upstream of the forward calorimeter front face (fig. 1). Interactions in the target were selected with a scintillator hodoscope/silicon pad system.

We measured the total energy deposited in the calorimeters for events where interactions in the target occur at a beam intensity of $10^{5}$ particles per second. We also selected events by triggering on cases where the secondary particles carry a minimum amount of energy in the plane perpendicular to the beam axis (transverse energy $E_{T}$ ), in order to study the influence of enlarging the area where the particles deposit their energy. The energy resolution was found to be about a factor of 2 worse than for single particles, and depends weakly on the $E_{T}$ threshold. An obvious contribution to this effect comes from the fact that in order to obtain the total signal, pedestal subtraction has to be applied for an increasing number of channels. The pedestal values for uranium calorimeters contain, apart from the ADC pedestal, contributions from uranium noise, of which the light production in our standard $130 \mathrm{~ns}$ gate time corresponds to $100 \mathrm{MeV}$ on an average for a hadronic calorimeter section. The fluctuation in this number is such that adding up 432 channels (the forward calorimeter part) gives an uncertainty of $0.8 \mathrm{GeV}$. If the whole calorimeter is read out ( 2860 channels) this number increases to $2.1 \mathrm{GeV}$, implying a contribution of $0.15 / \sqrt{\mathrm{E}}$ to $\sigma / \mathrm{E}$ at $200 \mathrm{GeV}$. This is not negligible compared to the total energy resolution of $0.34 / \sqrt{\mathrm{E}}$ measured for single-particle detection. For this reason alone, a consequence of the radioactivity of ${ }^{238} \mathrm{U}$, the best achievable energy resolution cannot be better than $0.37 / \sqrt{\mathrm{E}}$ when the whole calorimeter has to be read out to detect the signal. In fact, the measured energy resolutions are worse than that and so the effects mentioned before certainly contribute to the degradation of performance as well. In particular the $\mathrm{Cu} /$ scintillator modules severely suffered from radiation damages before they were installed in HELIOS and put in an inert atmosphere, so that their multiparticle resolution is strongly degraded [2].

The calculation of energy resolution from a signal distribution requires that the total energy carried by the particles that enter the apparatus is known and constant. 


\subsection{Performance of the Energy Flow Logic}

The EFL is an integral part of the HELIOS trigger facilities. By providing fast, on-line information on the transverse energy, the missing energy, or the missing transverse momentum of an event, it makes it possible to select extremely rare events at the trigger level. In this section we just show a few examples to illustrate to what extent this logic provides the correct information. This can be judged by comparing the on-line values, obtained from the fast electronics of the EFL, with the reconstructed off-line values that come from the analysis of all the detailed charge ADC information. A useful quantity in evaluating the comparison between the FADC trigger signal and the corresponding charge ADC (CADC) sum is the asymmetry (FADC - CADC)/(FADC + CADC). Two of these asymmetries are shown in fig. 22 , for a $300 \mathrm{GeV} \pi^{-}$beam incident on a $5 \% \mathrm{~Pb}$ target. The threshold in $\mathrm{E}_{\mathrm{T}}$ was set at $30 \mathrm{GeV}$. Figure $22 \mathrm{a}$ shows the asymmetry for the total energy and fig. $22 \mathrm{~b}$ the asymmetry for $\mathrm{E}_{\mathrm{T}}$. In both cases the asymmetries are centred on the origin, showing that the trigger and off-line energy scales are in good agreement. The r.m.s. widths of the distributions are $2.3 \%$ and $5 \%$ for $\mathrm{E}_{\text {tot }}$ and $\mathrm{E}_{\mathrm{T}}$, respectively. These figures show that the trigger energy follows the summed charge ADC energy to within the fluctuations of the uranium noise (see previous section).

Of great importance for the physics analysis is the sharpness of the threshold in $\mathrm{E}_{\mathrm{T}}$. In fig. 23 the FADC trigger signal and the corresponding charge ADC sum are shown for $300 \mathrm{GeV} \pi^{-}$. The off-line charge ADC values show that the convolution with the fluctuations of the uranium noise signal introduces a corresponding inefficiency around the nominal $30 \mathrm{GeV}$ threshold energy.

The EFL was designed to cope both with proton beams up to $450 \mathrm{GeV}$ and ion beams up to $200 \mathrm{GeV}$ per nucleon. This is achieved by suitable attenuation factors in the $\Sigma_{2}$ summing units. Although most of the fine tuning of the amplifier gains was done using $p$ and $\pi$ beams, the calibration has proved to be accurate over the much wider energy range provided by ${ }^{16} \mathrm{O}$ beams (cf. fig. 20). As an example of the EFL response at $3.2 \mathrm{TeV}$, fig. $24 \mathrm{a}$ shows the correlation between the FADC signal and the corresponding charge ADC sum for the transverse energy of the 'box' calorimeter. A maximum transverse energy of the order of $170 \mathrm{GeV}$ is recorded, a factor 4 times as large as with proton beams. The difference between the two signals (fig. 24b) is completely explained by the uranium noise fluctuations. Figure 25 shows the transverse-energy spectrum obtained on line for the same set of data, which were obtained by triggering on four different transverse-energy thresholds, at $40,73,106$, and $139 \mathrm{GeV}$. On the figure, these four classes of events are clearly shown, along with minimum-bias events and random triggers, all classes being suitably downscaled in order to arrive at an optimum trigger composition.

\section{CONCLUSIONS}

We have described the calorimeter of the HELIOS experiment, the calibration procedures, and the results of the measurements made for determining the performance of this crucial part of the detector. We extensively discussed the problems arising when calibrating a longitudinally segmented calorimeter, and the meaning of longitudinal shower information. The calorimeter response to single particles is linear with the energy to within $2 \%$, over the energy range $8 \mathrm{GeV}$ to $200 \mathrm{GeV}$. The energy resolution $\sigma / \mathrm{E}$ for detecting single electrons and pions scales with $1 / \sqrt{\mathrm{E}}$ and was found to be $(0.215 \pm 0.007) / \sqrt{\mathrm{E}}$ and $(0.337 \pm 0.013) / \sqrt{\mathrm{E}}$, respectively $(\mathrm{E}$ in $\mathrm{GeV})$. For the signal ratio $\mathrm{e} / \pi$ a value of $0.984 \pm 0.006$ was measured with a $130 \mathrm{~ns}$ gate. The value of $\mathrm{e} / \mu$ is strongly dependent on the energy; we used the experimental data to determine the energy-independent quantity $\mathrm{e} / \mathrm{mip}$, for which a value of $0.70 \pm 0.05$ was found.

The light signals generated by the shower extend over quite a long period, about $1 \mu \mathrm{s}$. This is partly due to fluorescence phenomena, and partly to the detection of $\gamma$ 's from captured neutrons, a slow process that only plays a role for hadron detection, and therefore makes the $\mathrm{e} / \pi$ ratio dependent on the signal integration time, as experimentally observed. 
The energy resolution for detecting multiple particles from interactions is found at $200 \mathrm{GeV}$ to be approximately a factor of 2 worse than that obtained for single particles. Part of this effect is due to the larger uncertainties inherent in the subtraction of contributions of uranium noise and inhomogeneities in the detector.

The Energy Flow Logic that generates the trigger information on physics quantities relevant to the goals of the experiment performed as expected. The resolution between the trigger energy and the summed charge ADC energy is explained by the uranium noise fluctuations and therefore is much better than the overall calorimeter resolution.

\section{Acknowledgements}

The work described in this paper would not have been possible without the help of many people. We benefited greatly from the presence of an excellent data-taking infrastructure, to which many of our HELIOS colleagues contributed. Outstanding technical assistance in the (re)construction and installation of the calorimeters was provided by J.C. Berset, M. Burns, K. Bussmann, B. Couprie, G. Delavallade, M. Gibson, J. Hiddleston, C. Marin and J.P. Vanuxem. We are also grateful for the support of the Natural Sciences and Engineering Research Council of Canada, the Science and Engineering Research Council of the United Kingdom, and the Department of Energy of the USA. 


\section{REFERENCES}

[1] T. Åkesson et al., Nucl. Instrum. Methods A241 (1985) 17.

[2] Y. Sirois and R. Wigmans, Nucl. Instrum. Methods A240 (1985) 262.

[3] H. Brueckmann and H. Kowalski, The dependence of calorimeter responses on the gate width, DESY, Hamburg, ZEUS Int. Note 86/026 (1986).

[4] R. Wigmans, On the energy resolution of uranium and other hadron calorimeters, preprint CERN-EP/86-141, to be published in Nucl. Instrum. Methods.

[5] H. Abramowicz et al., Nucl. Instrum. Methods A180 (1981) 429.

[6] C.W. Fabjan, Calorimetry in high-energy physics, preprint CERN-EP/85-54 (1985), and references therein.

[7] J.F. Janni, At. Data Nucl. Data Tables 27 (1982) 147.

[8] W. Lohmann, R. Kopp and R. Voss, Energy loss of muons in the energy range $1 \mathrm{GeV}$ to $10000 \mathrm{GeV}$, CERN 85-03 (1985).

[9] H. Brueckmann, Hadron calorimetry-puzzle of physics, in Proc. Workshop on Compensated Calorimetry, Pasadena, 1985, CALT 68-1305.

[10] C. Leroy, Y. Sirois and R. Wigmans, Nucl. Instrum. Methods A252 (1986) 4. 


\section{Table 1}

Results on energy resolution, signal linearity, and $e / \pi$, including systematic errors

( $E$ in the energy resolution represents peak values)

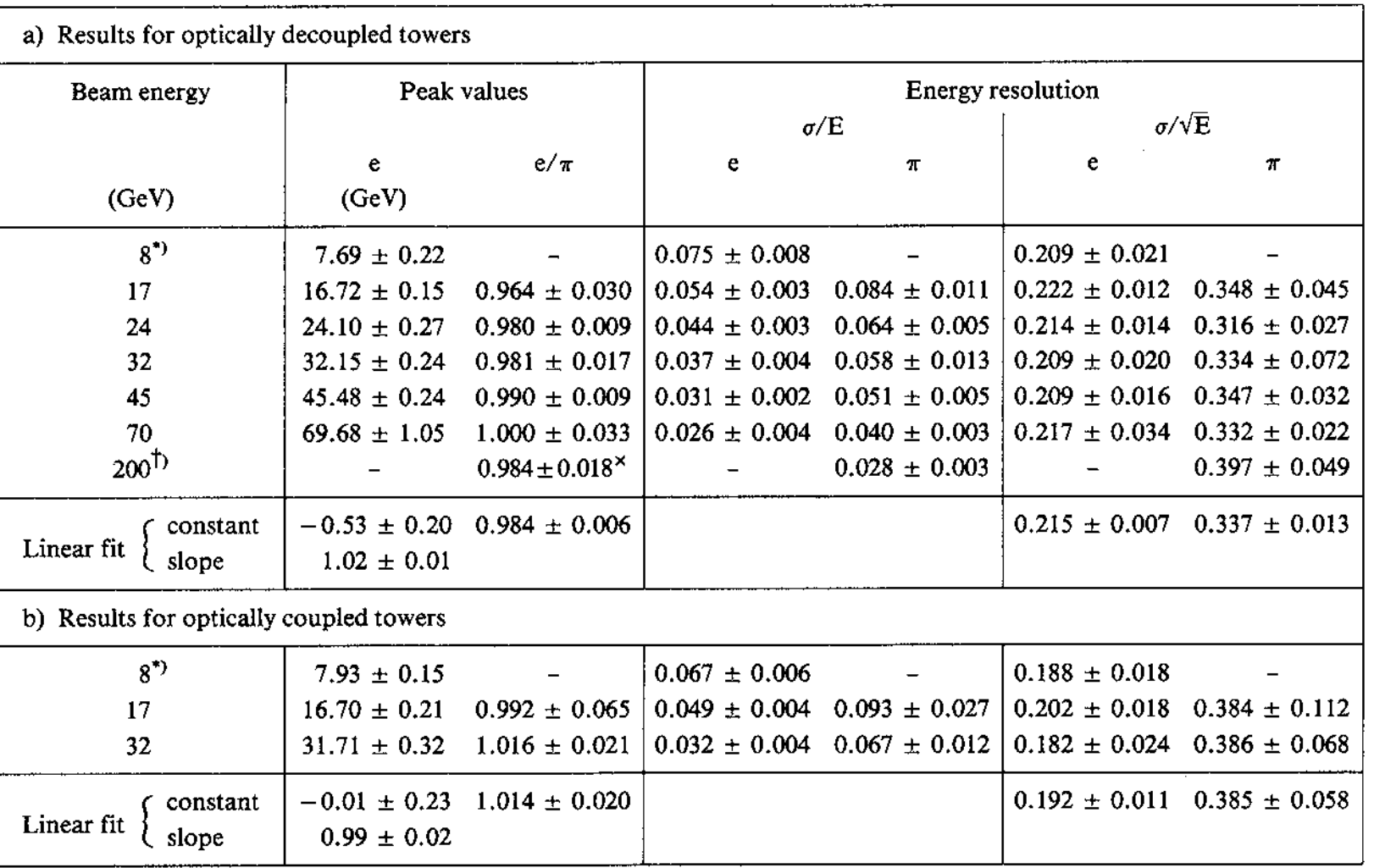

*) No pions available at this energy.

$\dagger$ ) No electrons available at this energy.

$\times$ ) Assuming electron signal linearity. This result is not included in the fit. 


\section{Figure captions}

Fig. 1 The HELIOS calorimeter layout. The target is surrounded by a box consisting of a calorimetrized dipole magnet (MAGCAL), U/scintillator and $\mathrm{U} / \mathrm{Cu} /$ scintillator modules. $\mathrm{The} \mathrm{Cu} /$ scintillator modules occupying the corners of the box are not shown. An external spectrometer (also not shown) views the target through the $20 \mathrm{msr}$ slit.

Fig. 2 Isometric view of one calorimeter module. The enlargement shows the arrangement of the optical readout and the sampling for the electromagnetic and hadronic sections.

Fig. 3 A scintillator plate used in the optically decoupled towers. Reflectors of aluminized Mylar are mounted inside the slits.

Fig. 4 Layout of the set-up for the optical measurements.

Fig. 5 The signals detected by a WLS plate as a point light source is moved at a fixed distance from the WLS plane. The response for coupled and decoupled towers is shown.

Fig. 6 Cross-talk pattern resulting from the leakage of light produced in a decoupled tower to a neighbour. The measurement is the ratio of the signals from two adjacent WLS.

Fig. 7 Artist's view of the calorimetrized dipole magnet (MAGCAL).

Fig. 8 a) The splitting of the PM anode signal and the input stage to the first level summing.

b) Schematic representation of the cabling and summing layout for the component of the calorimeter surrounding the target.

c) The logical interconnection of the EFL digital units. Box, beam etc., represent the different calorimeter components.

Fig. 9 a) The $\sigma_{\mathrm{rms}} / \mathrm{E}$ of the signal distribution as a function of $\mathrm{B} / \mathrm{A}$ from the electron and pion measurements.

b) The e/ $\pi$ signal ratio as a function of $\mathrm{B} / \mathrm{A}$ at 17 and $32 \mathrm{GeV}$.

Fig. 10 a) The signal distribution in the middle tower of the first row $\left(4.2 \lambda_{i}\right)$ of the forward calorimeter for a beam of $45 \mathrm{GeV}$ particles.

b) The total signal distribution of all towers of the forward calorimeter $\left(8.5 \lambda_{i}\right)$ for a beam of $45 \mathrm{GeV}$ particles.

Fig. 11 The $\sigma / \sqrt{\text { ratio }} \mathrm{E}$ as a function of energy for single electrons (open squares) and pions (closed circles). The CDHS [5] results ( $\pi$ ) are shown for comparison: $\mathrm{Fe}$ /scintillator before (open circles) and after (crosses) off-line weighting.

Fig. 12 The e/ $\pi$ signal ratio as a function of energy.

Fig. 13 The linearity of the calorimeter response to pions with energy. The signal per unit of energy is given (closed circles) for the detection of pions in the energy range (17 $\left.\leq \mathrm{E}_{\pi} \leq 200 \mathrm{GeV}\right)$ and the observed values are compared with those from CDHS [5]: Fe/scintillator before (open circles) and after (crosses) off-line weighting.

Tig. 14 The muon signal distributions for $8,24,70$, and $200 \mathrm{GeV}$ in the $8.4 \lambda_{\mathrm{i}}$ of the forward calorimeter. The dashed line is drawn at the peak value of the $200 \mathrm{GeV}$ data.

Fig. 15 Apparent muon energy loss as a function of incident energy.

Fig. 16 a) Contribution to $\mathrm{dE} / \mathrm{dx}$ as a function of the muon energy in uranium and, b) comparison between $\mathrm{dE} / \mathrm{dx}$ of uranium (high-Z absorber) and that of plastic scintillator (low- $Z$ readout).

Fig. 17 The $\mu /$ e signal ratios with and without corrections into real mip/e signal ratios.

Fig. 18 a) Electron and pion signals as a function of the charge ADC gate width.

b) Electron and pion response as a function of the delay on the charge ADC gate.

Fig. 19 Effect of intensity on energy resolution. The resolution improves if the events taken in the first $0.5 \mathrm{~s}$ of the burst are discarded or corrected for.

Fig. $20{ }^{16} \mathrm{O}-\mathrm{W}$ interaction for a $3.2 \mathrm{TeV}$ oxygen incident beam $(200 \mathrm{GeV}$ per nucleon). The total energy resolution is $1.9 \%$. The centre of the energy distribution is at $3.15 \mathrm{TeV}$. 
Fig. 21 The leakage in hadron showering measured behind $4.2 \lambda_{\mathrm{i}}$ of $U /$ scintillator for $24,45,70$, and $200 \mathrm{GeV}$ incoming pions. The data are presented as the fraction of the events having a leakage energy above a given threshold.

Fig. 22 Asymmetry between flash ADC and charge ADC readouts for a $300 \mathrm{GeV}$ pion beam incident on a $5 \% \mathrm{~Pb}$ target: a) for $\mathrm{E}_{\text {tot }}$, and $b$ ) for $\mathrm{E}_{\mathrm{T}}$.

Fig. 23 The FADC trigger signal and the corresponding charge ADC sums are shown for a $300 \mathrm{GeV}$ pion beam and a threshold $\mathrm{E}_{\mathrm{T}}>30 \mathrm{GeV}$.

Fig. 24 a) Correlation between the FADC signal and the corresponding CADC sum for the transverse energy signals from the calorimeter 'box' (section 2.1).

b) Flash ADC - charge ADC difference corresponding to (a).

Fig. 25 Transverse energy spectrum obtained on line from trigger data. 


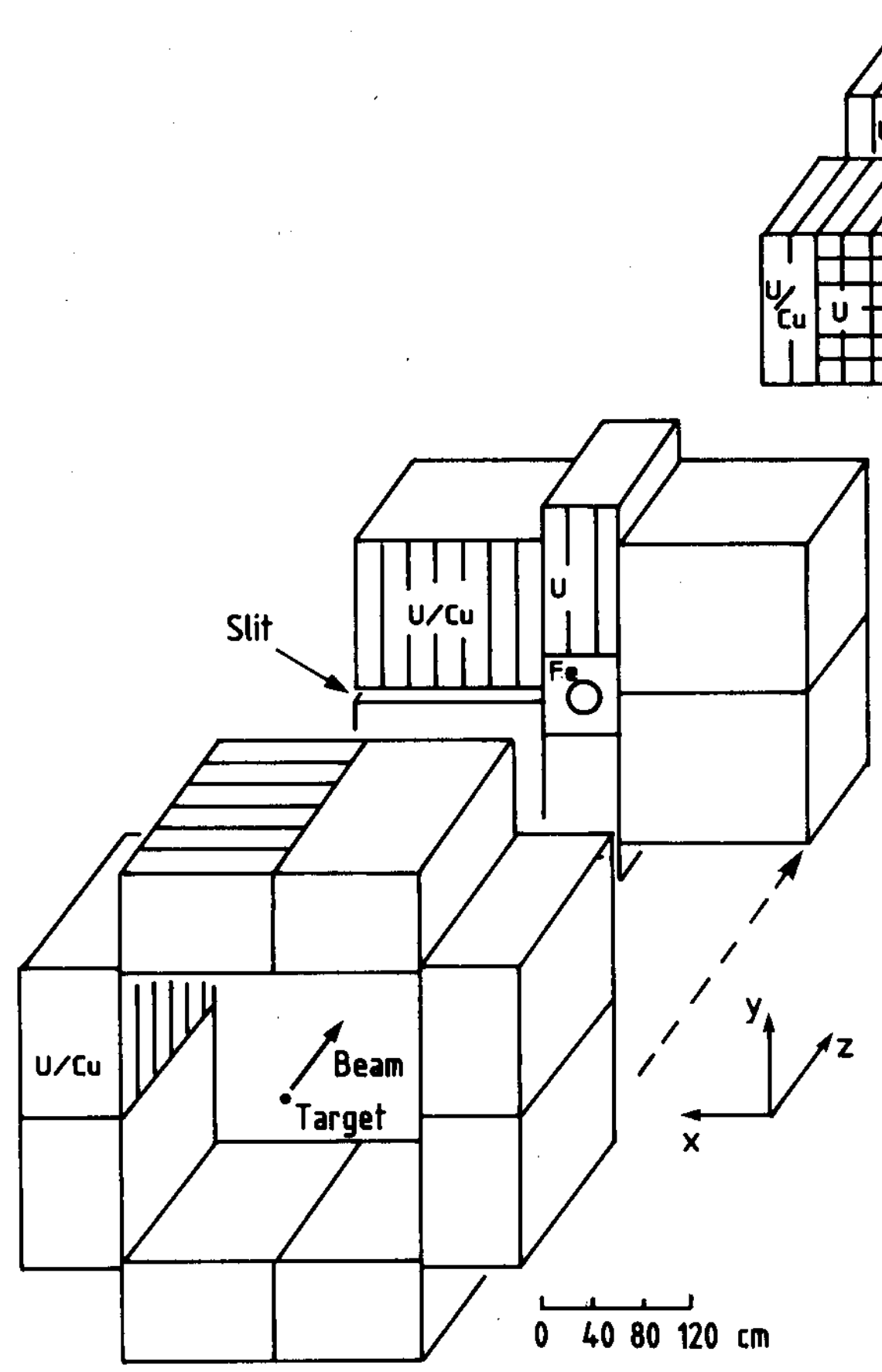

Fig. 1 


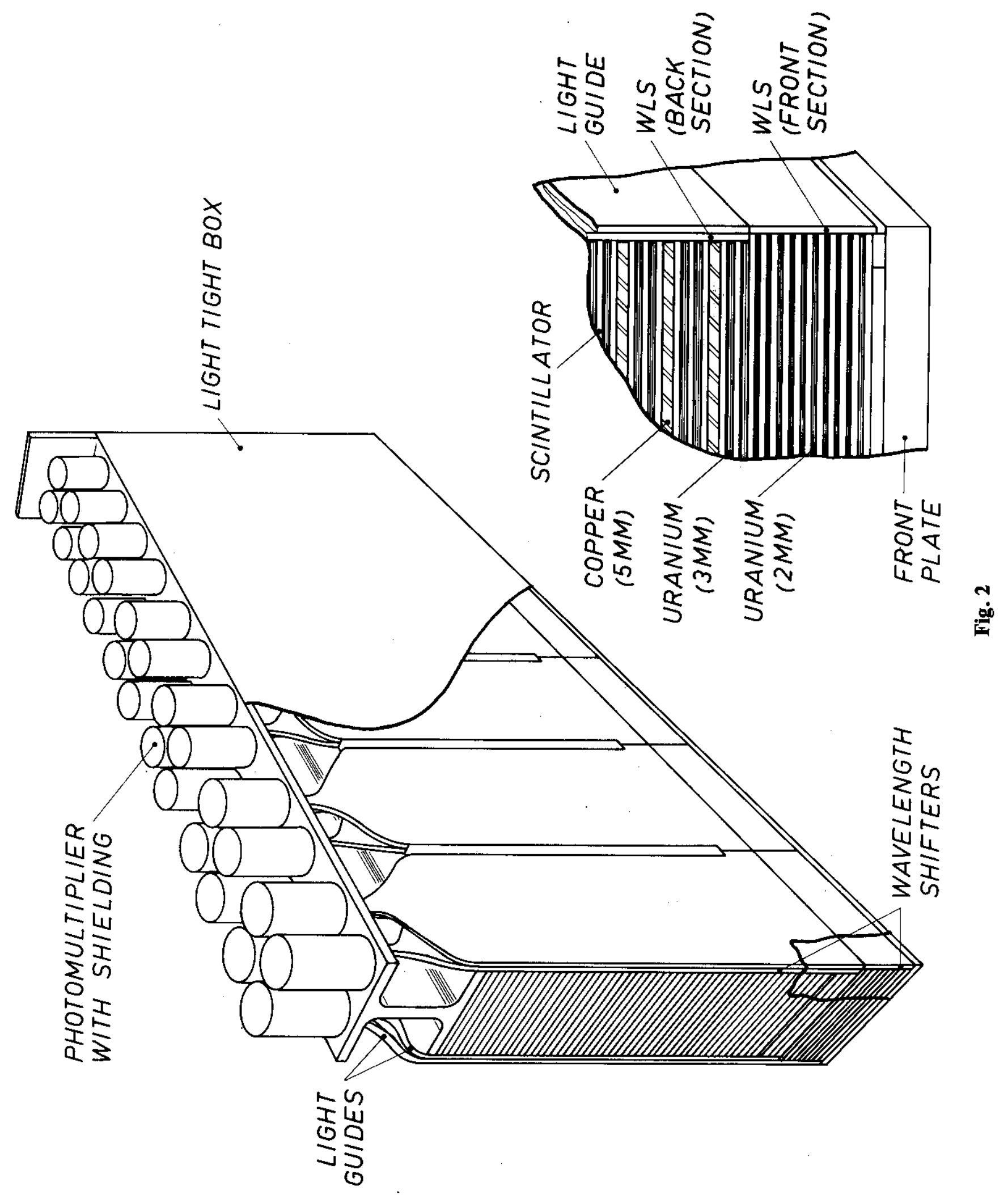




$$
\text { l }
$$




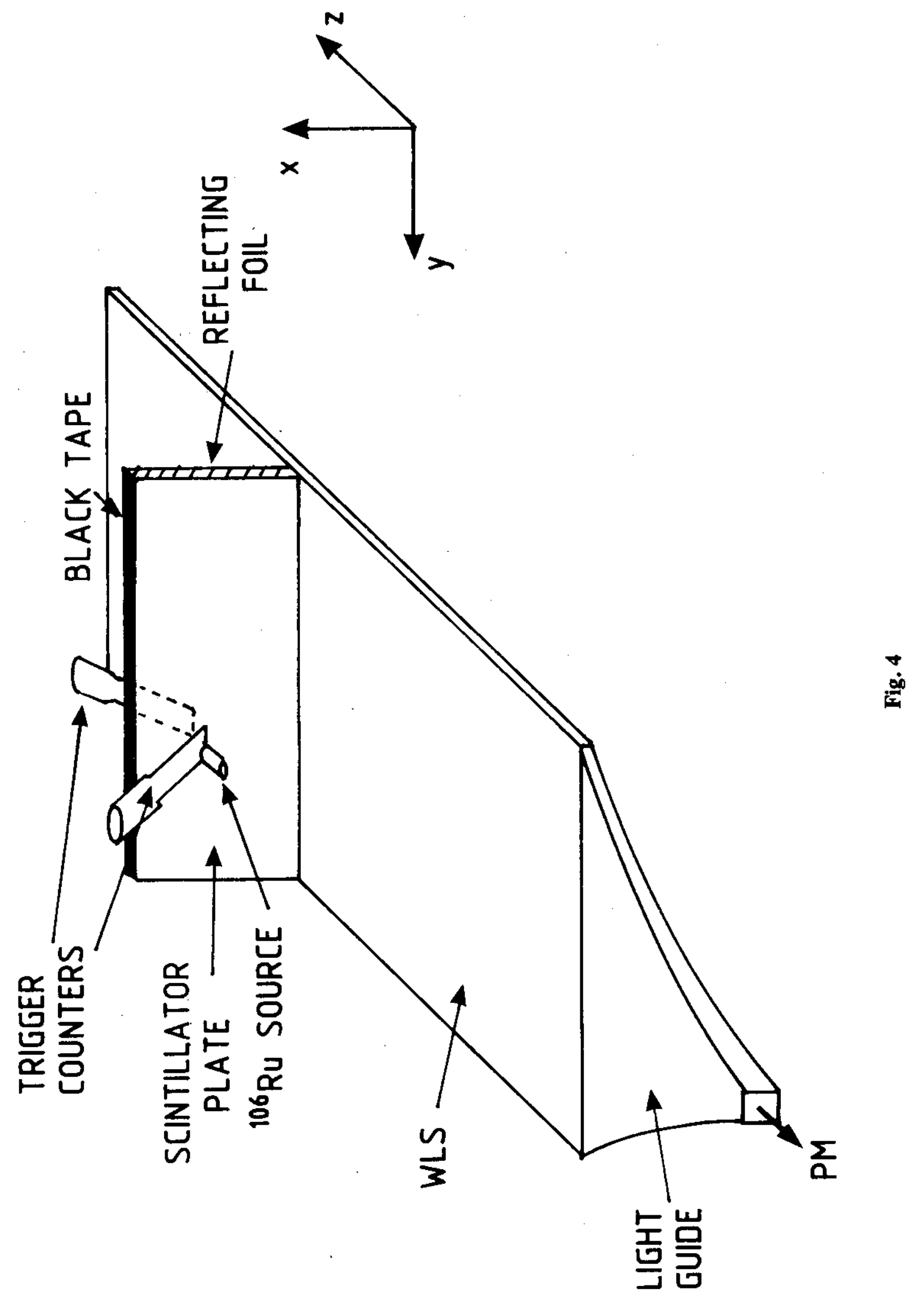




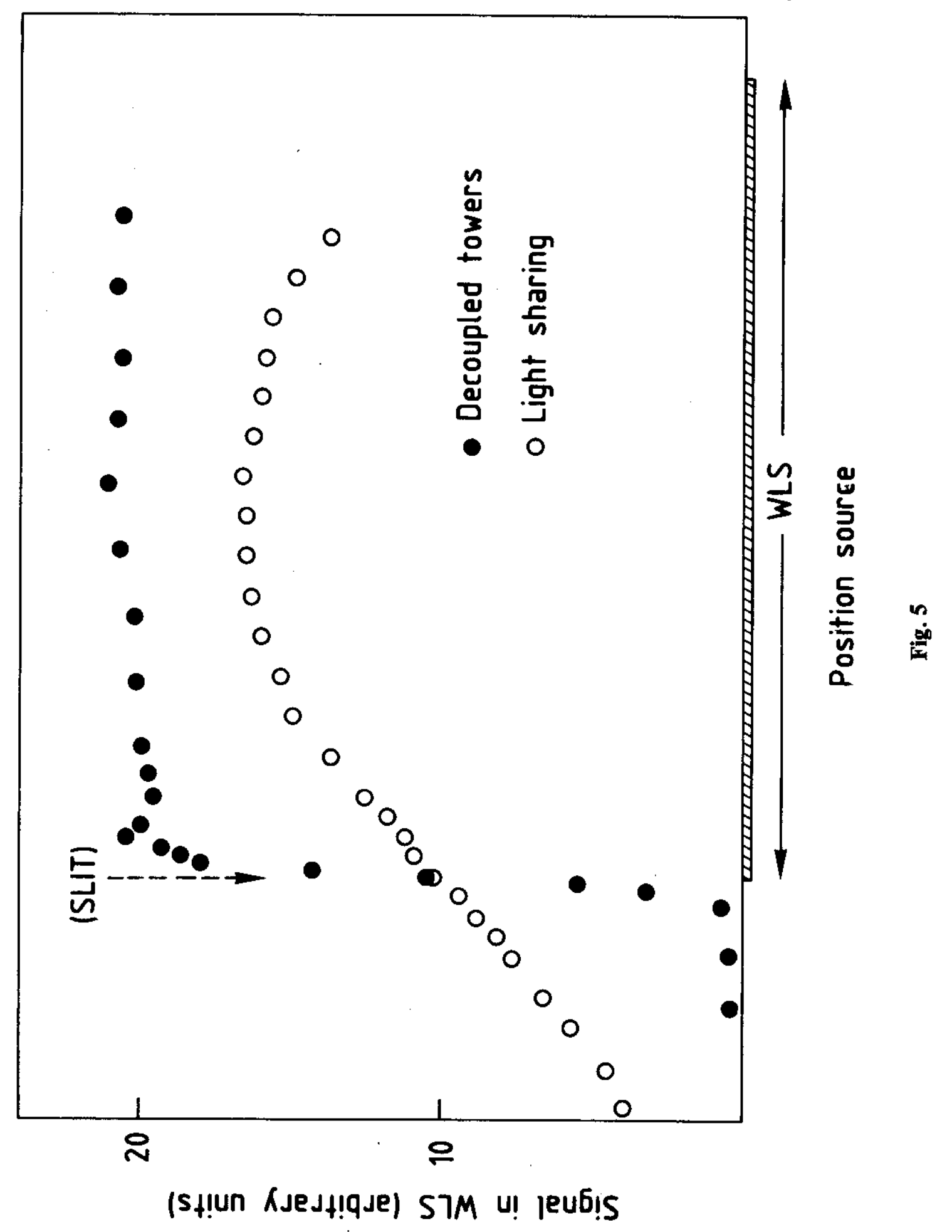



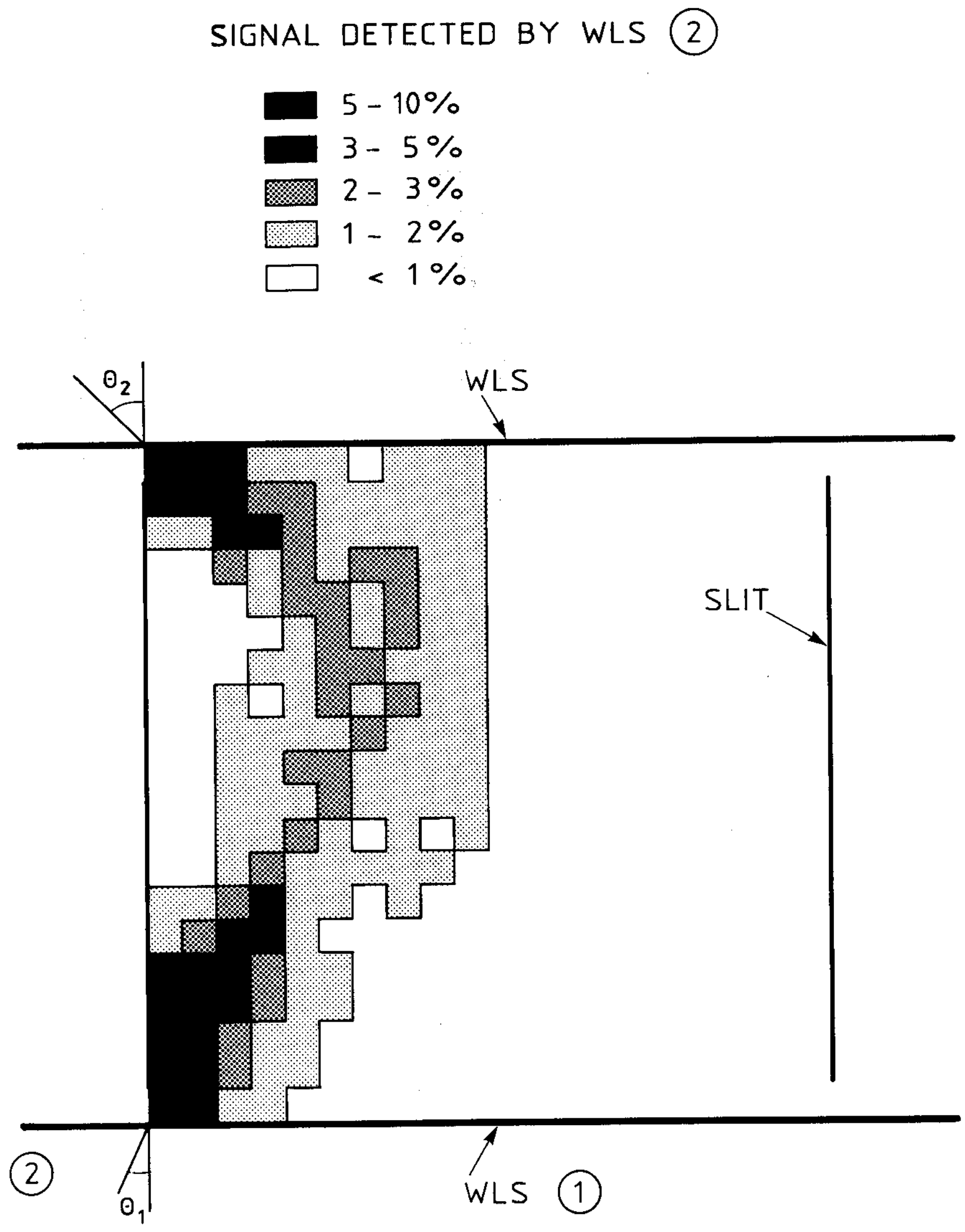

Fig. 6 


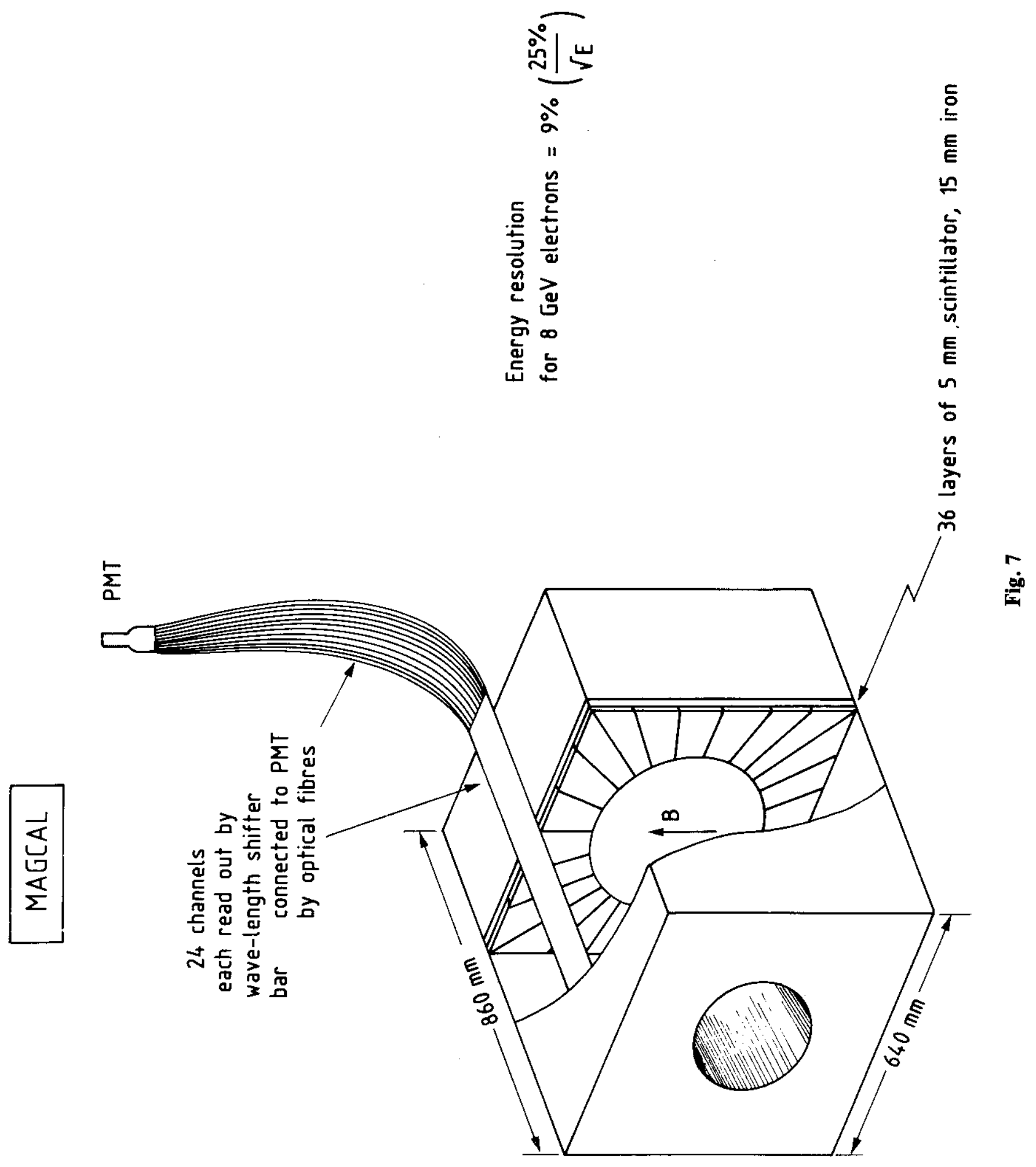




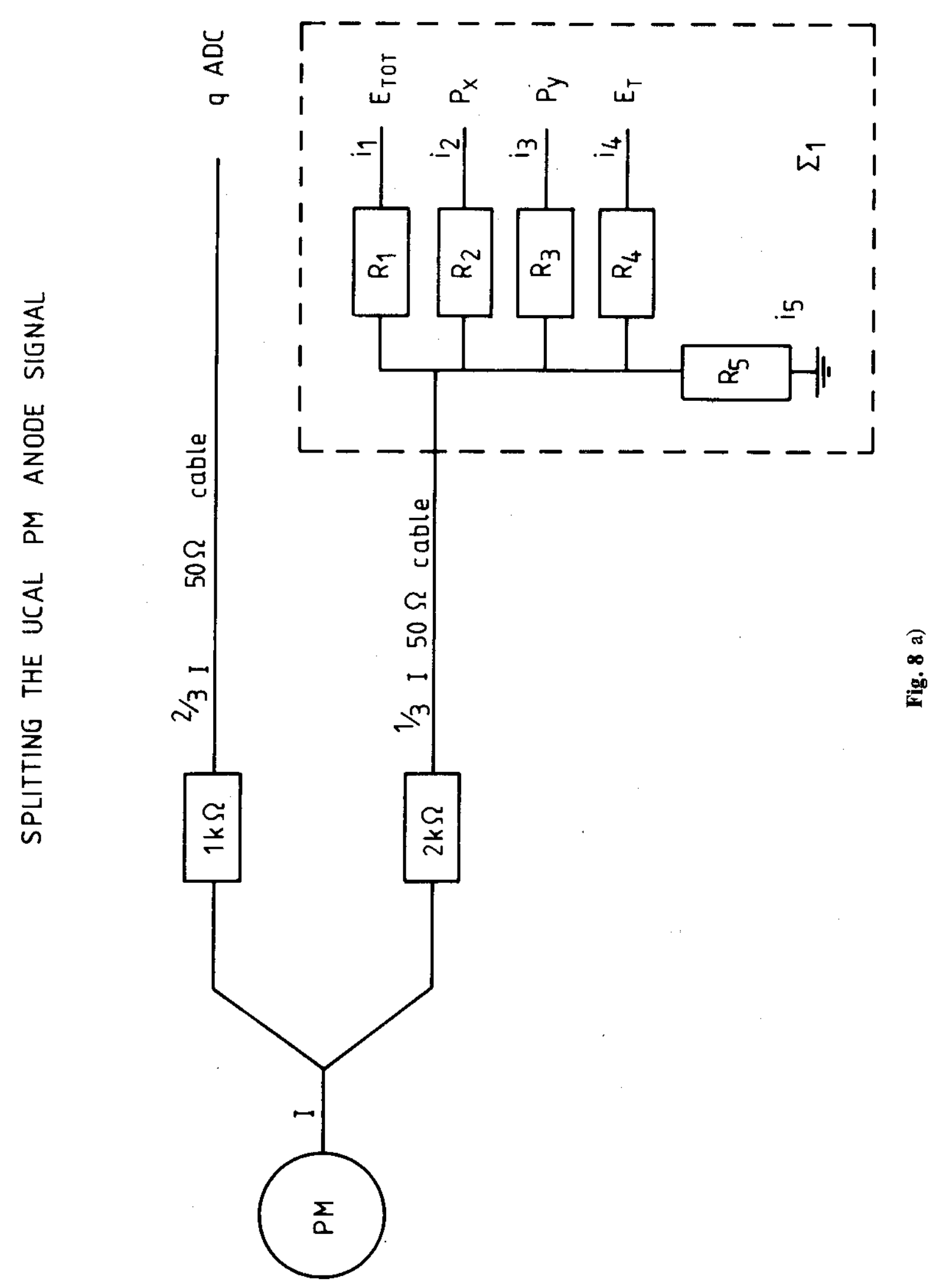




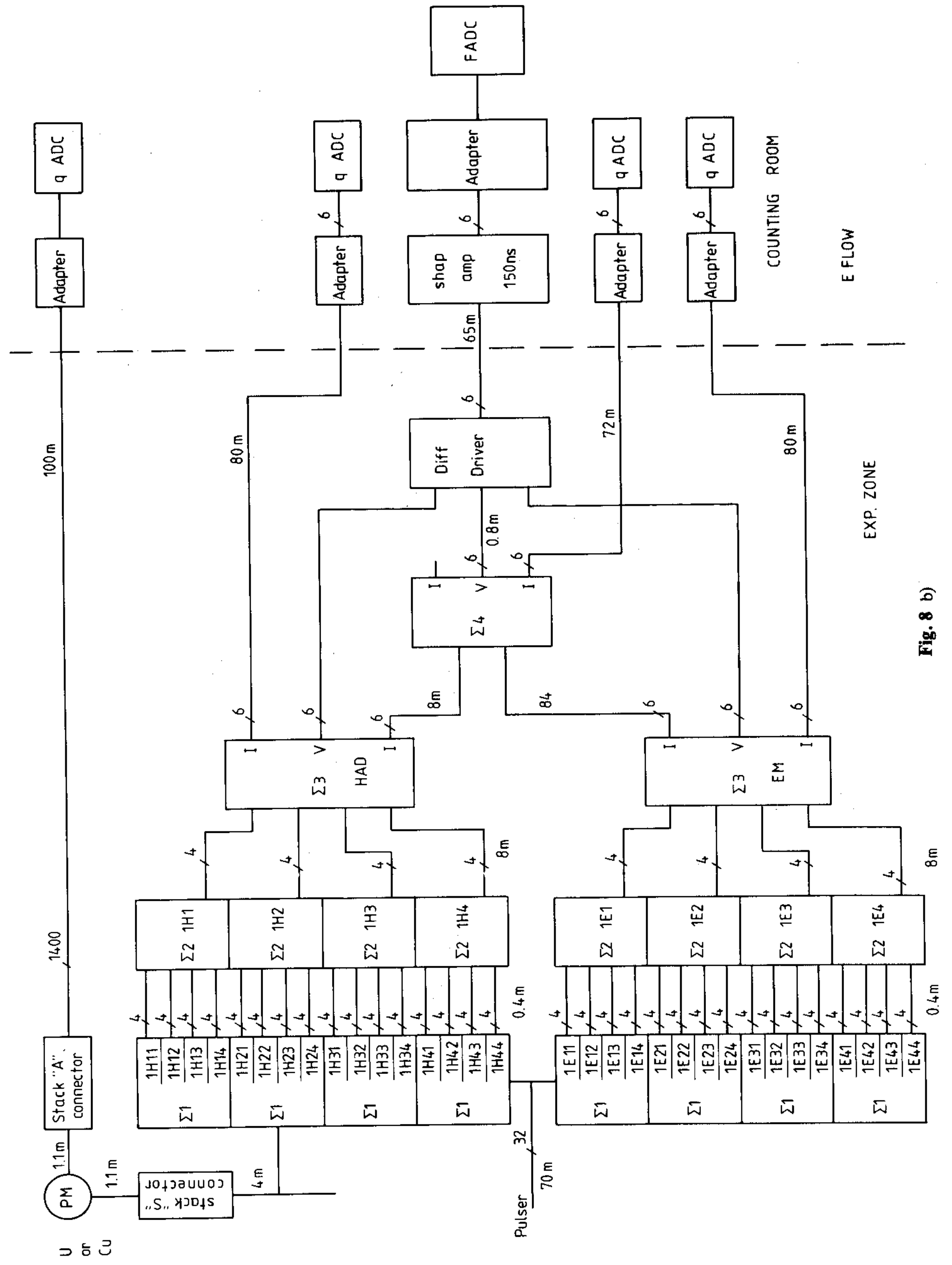




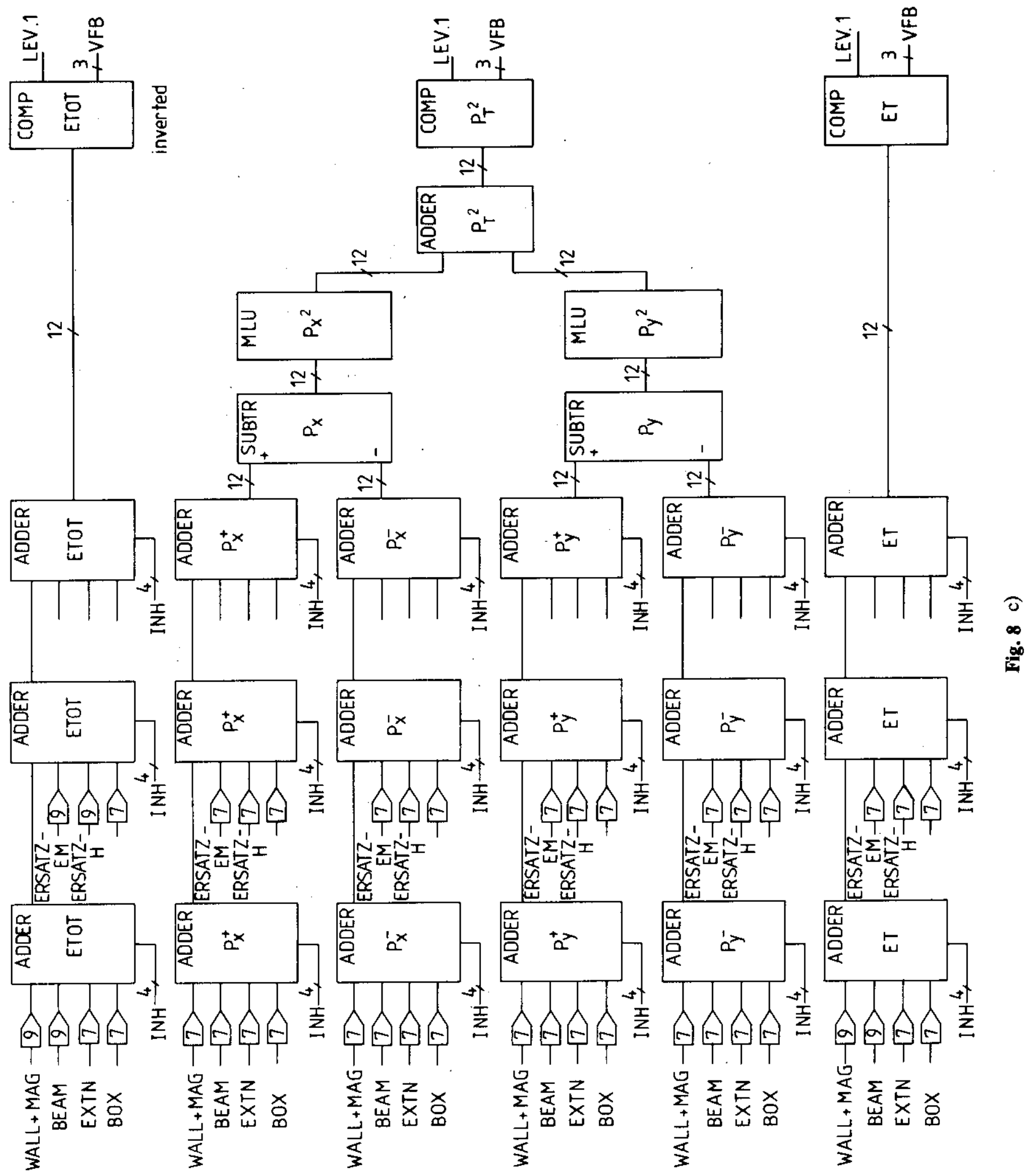




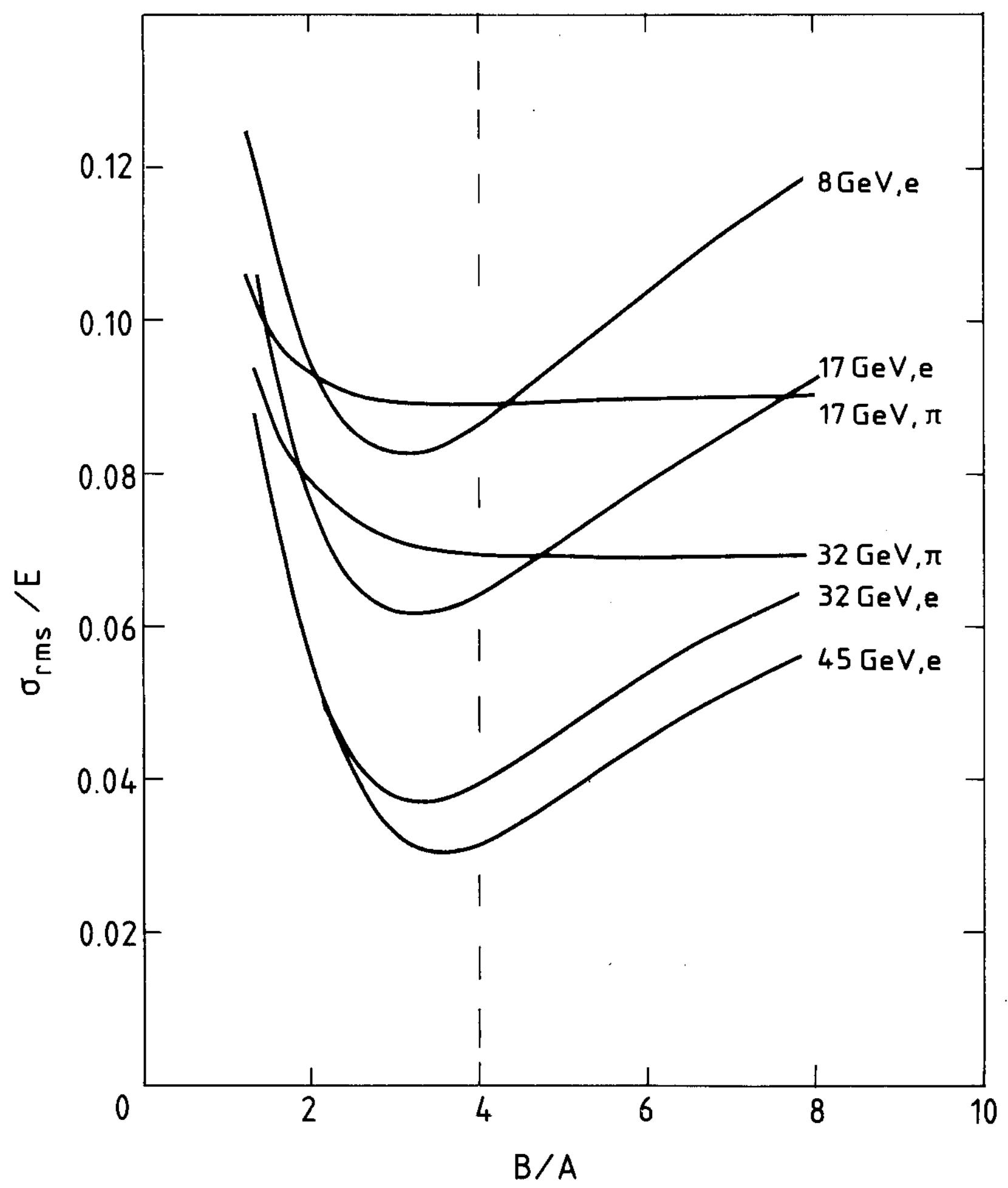

Fig. 9 a) 


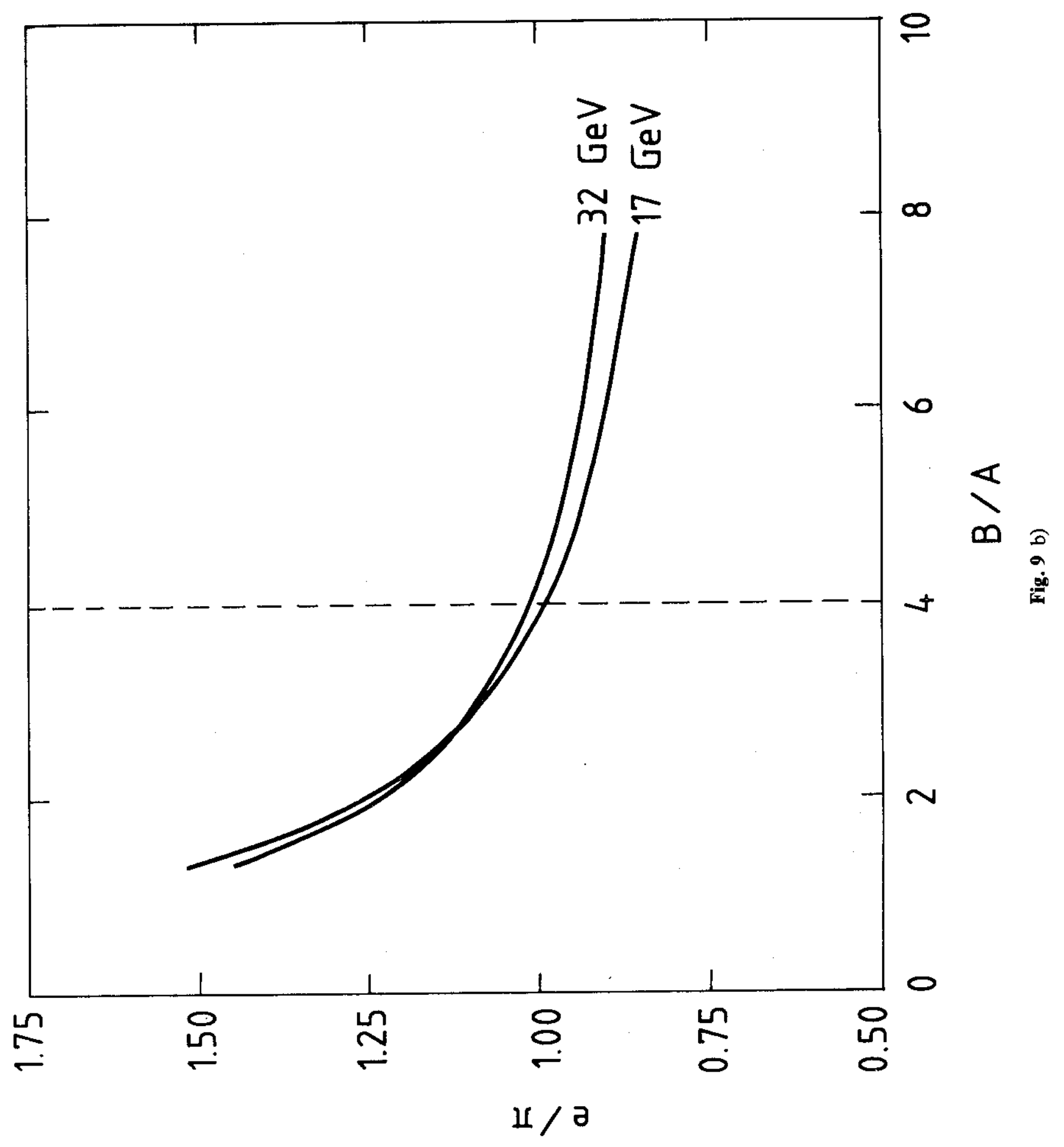



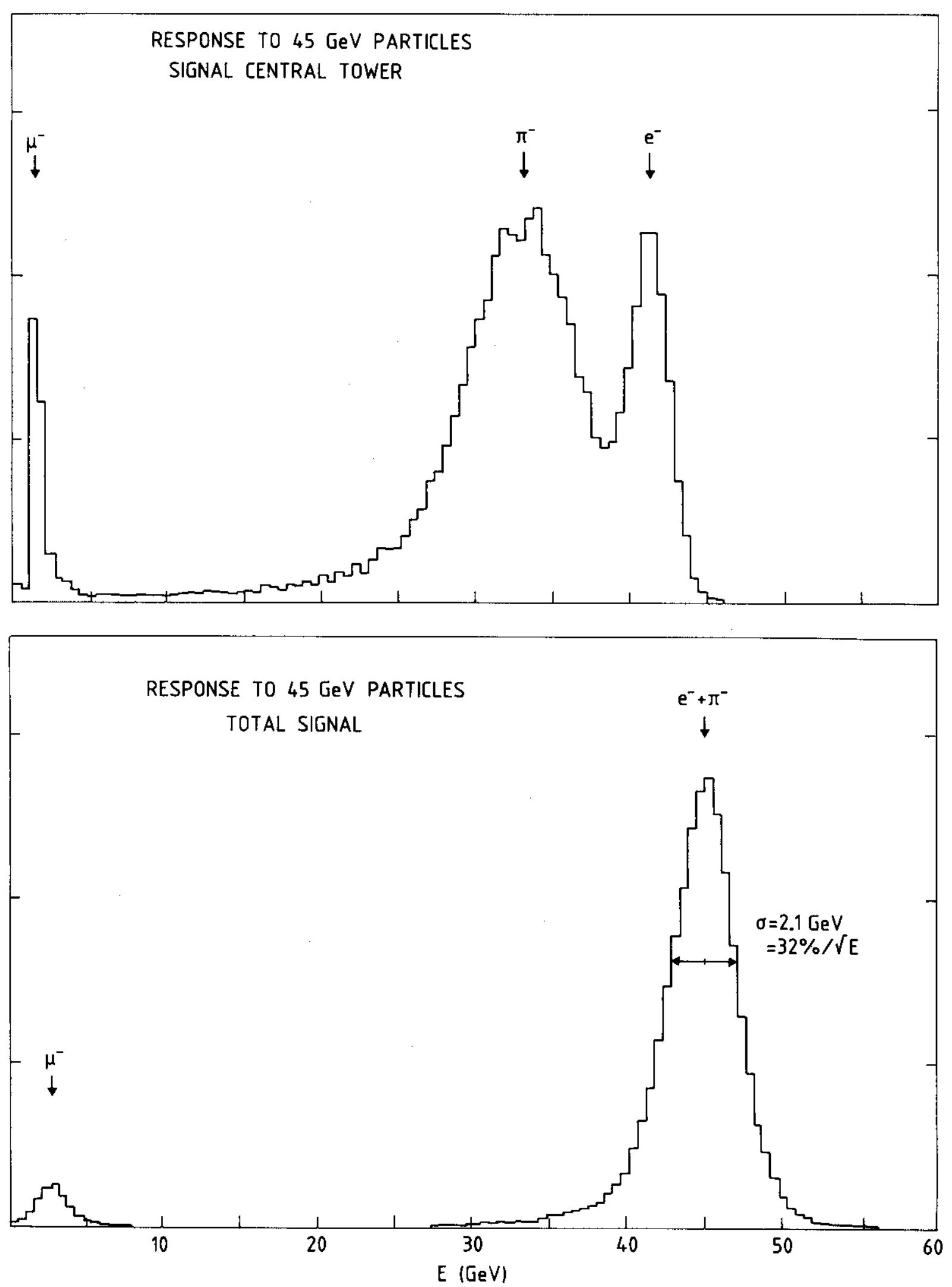

Fig. 10 


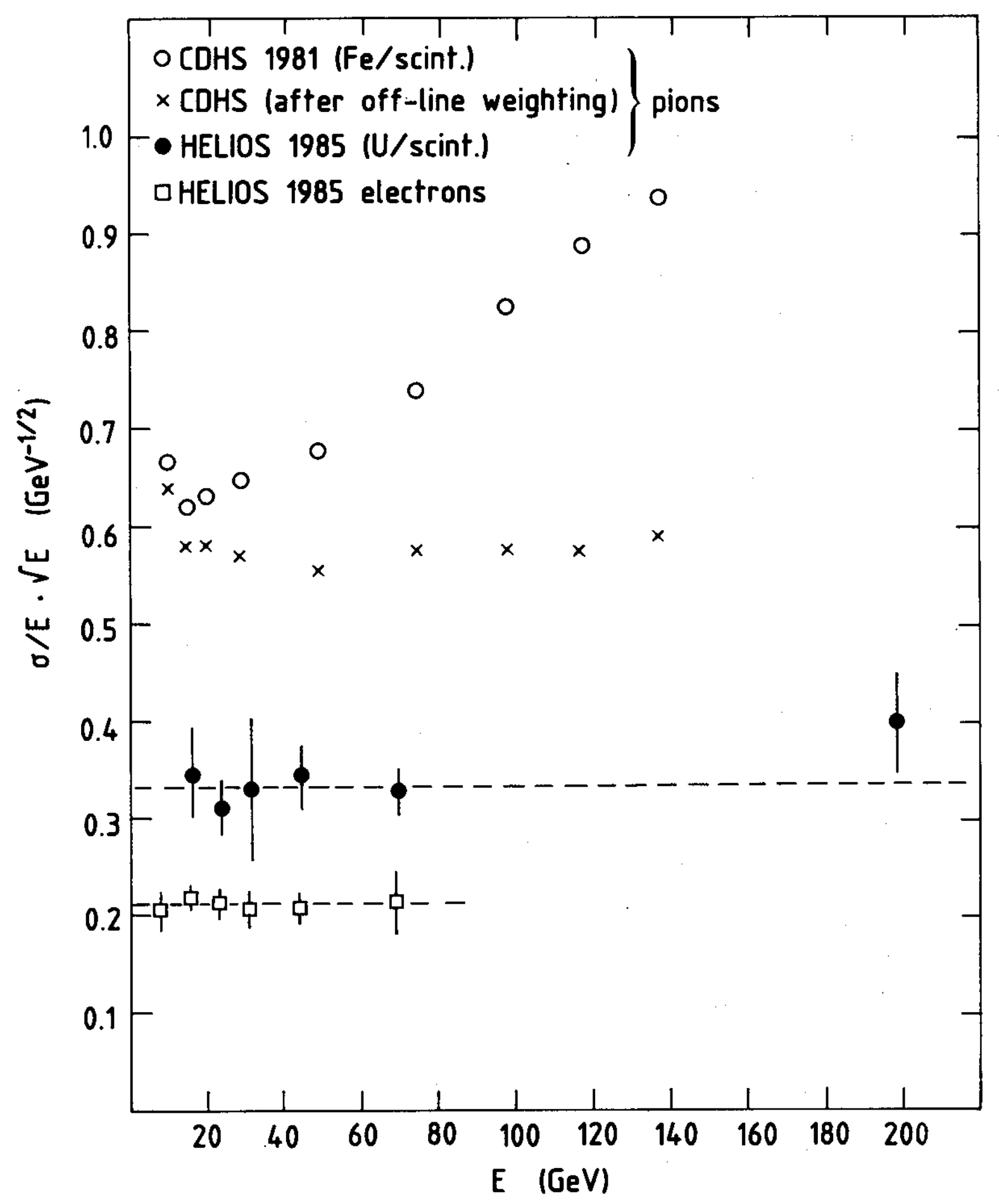

Fig. 11 


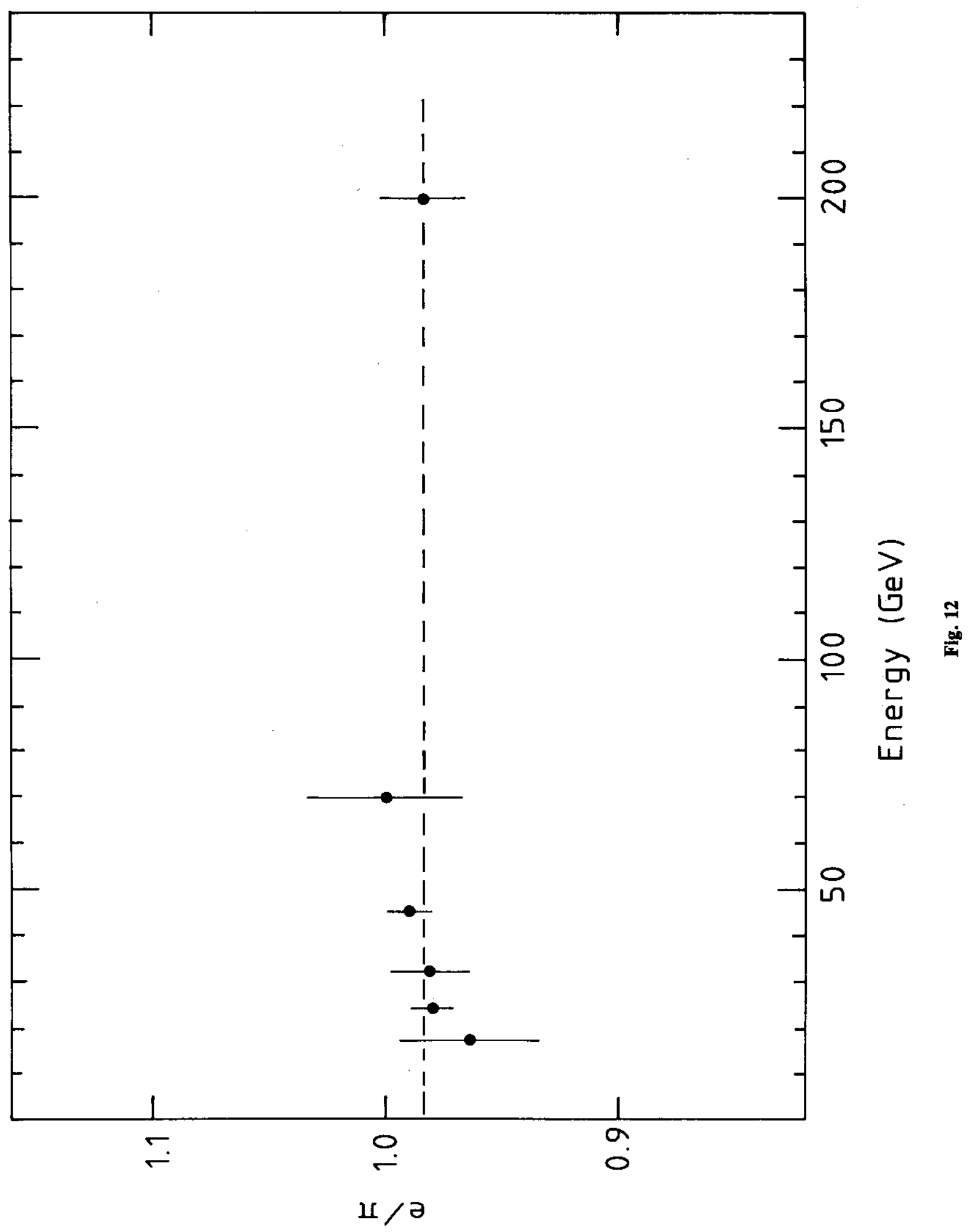




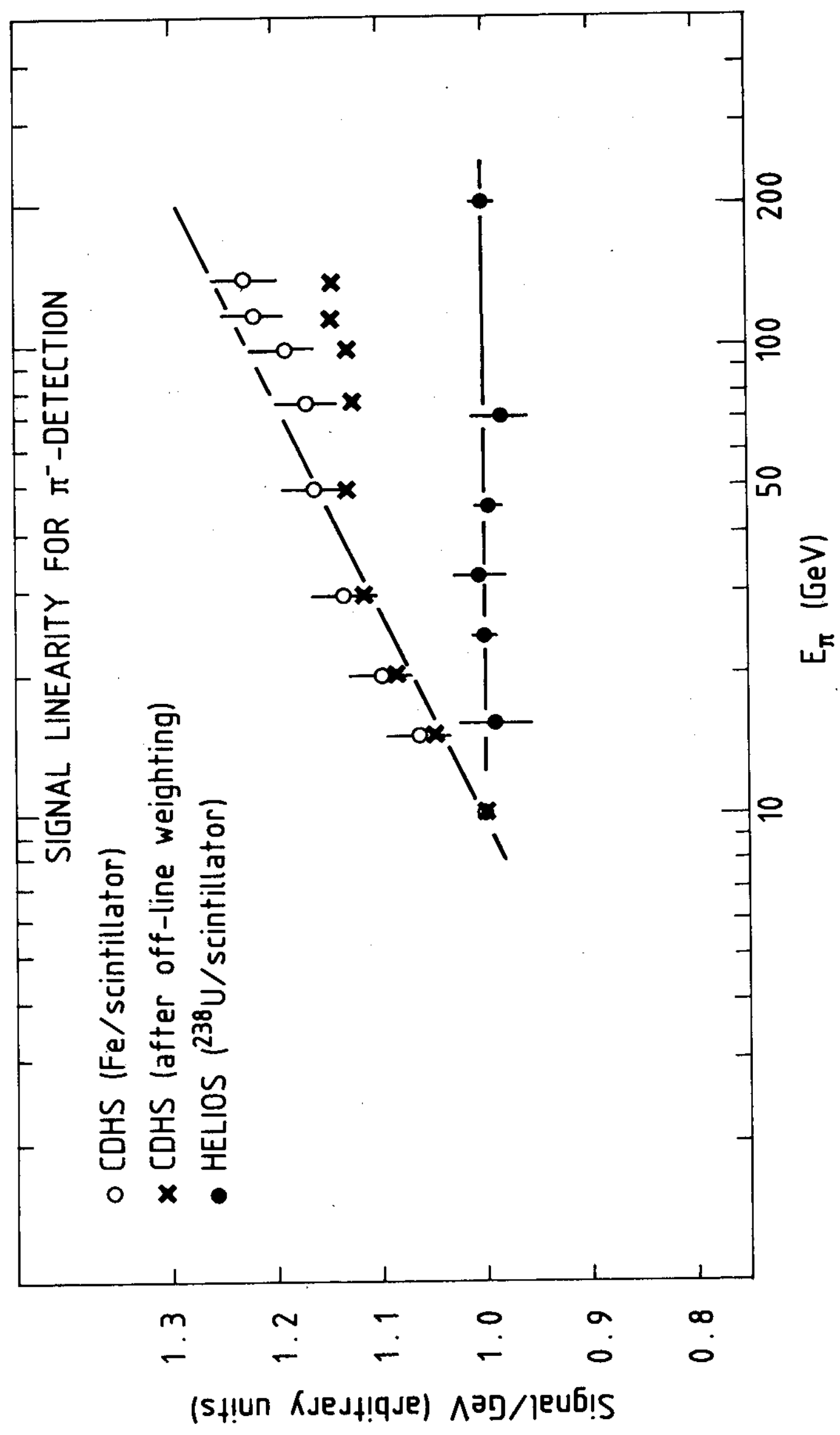

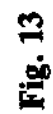




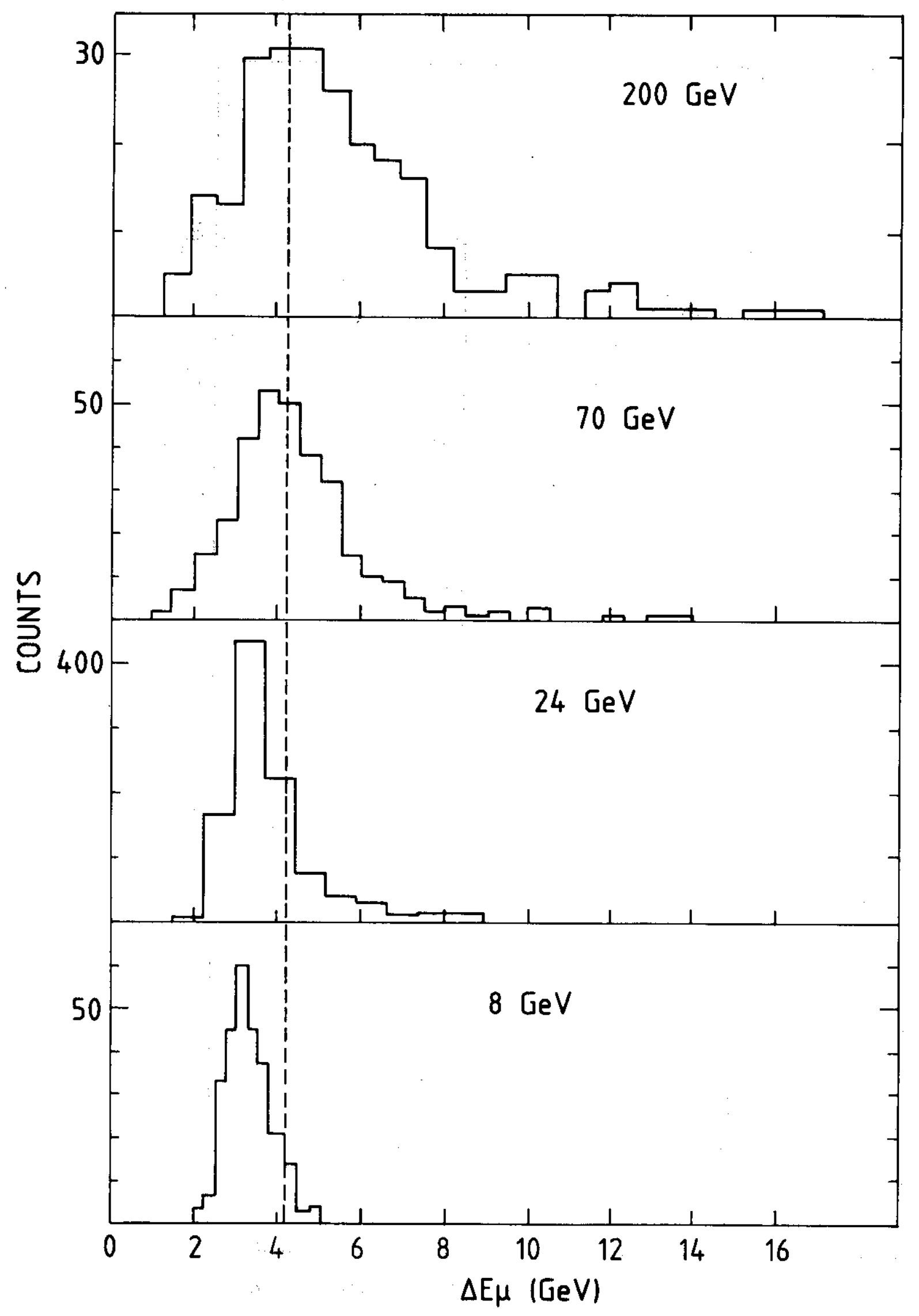

Fig. 14 


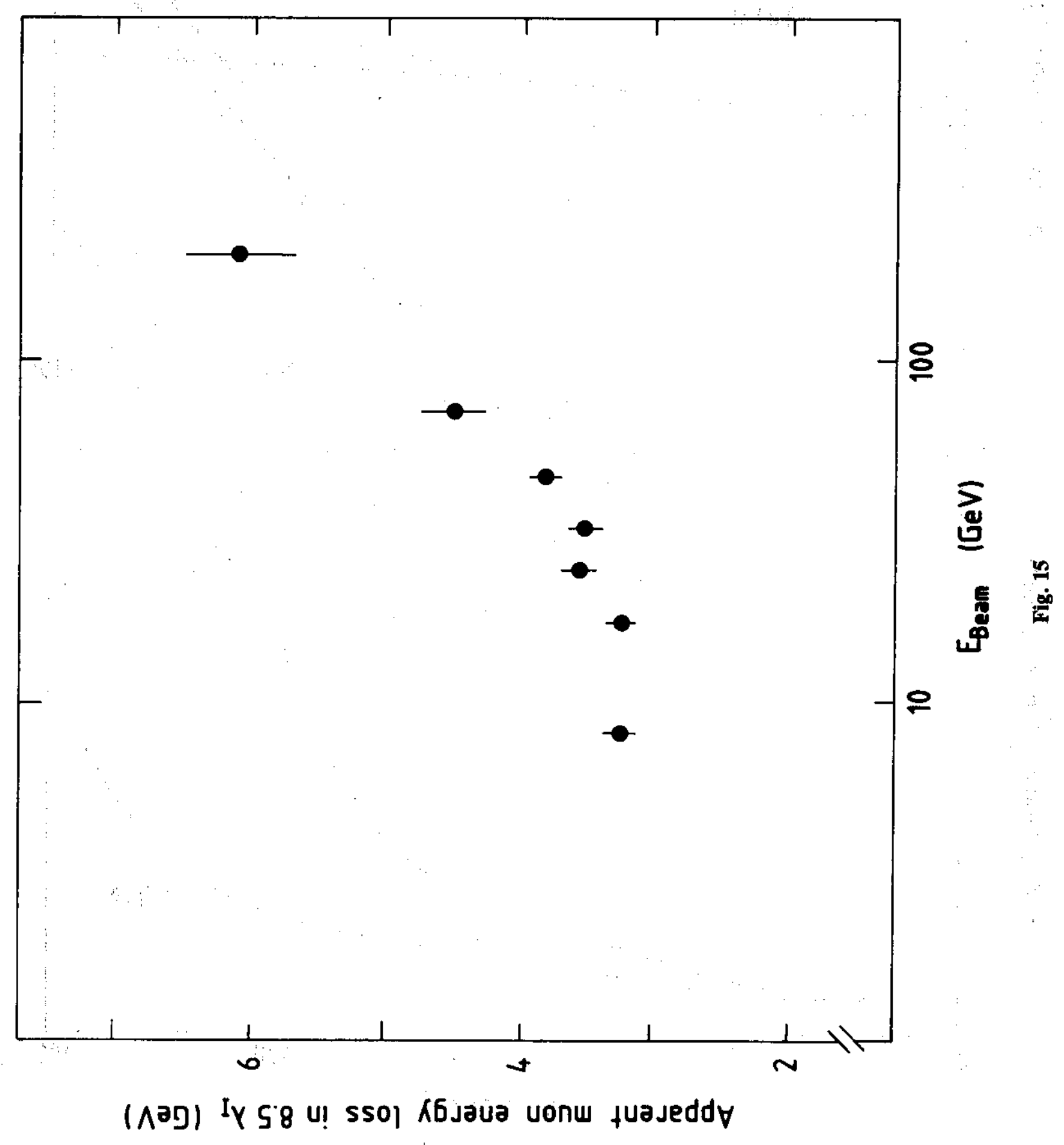



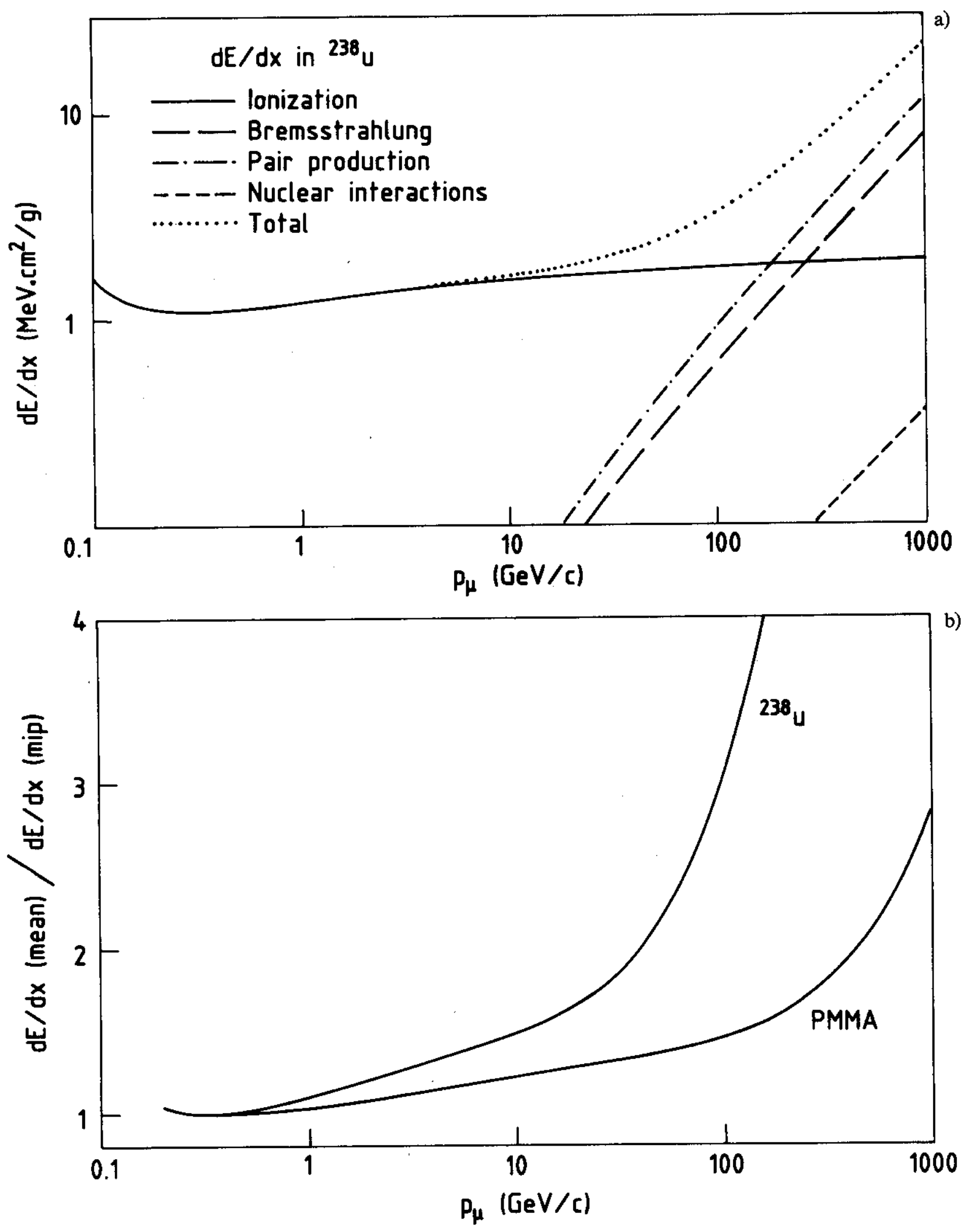

Fig. 16 


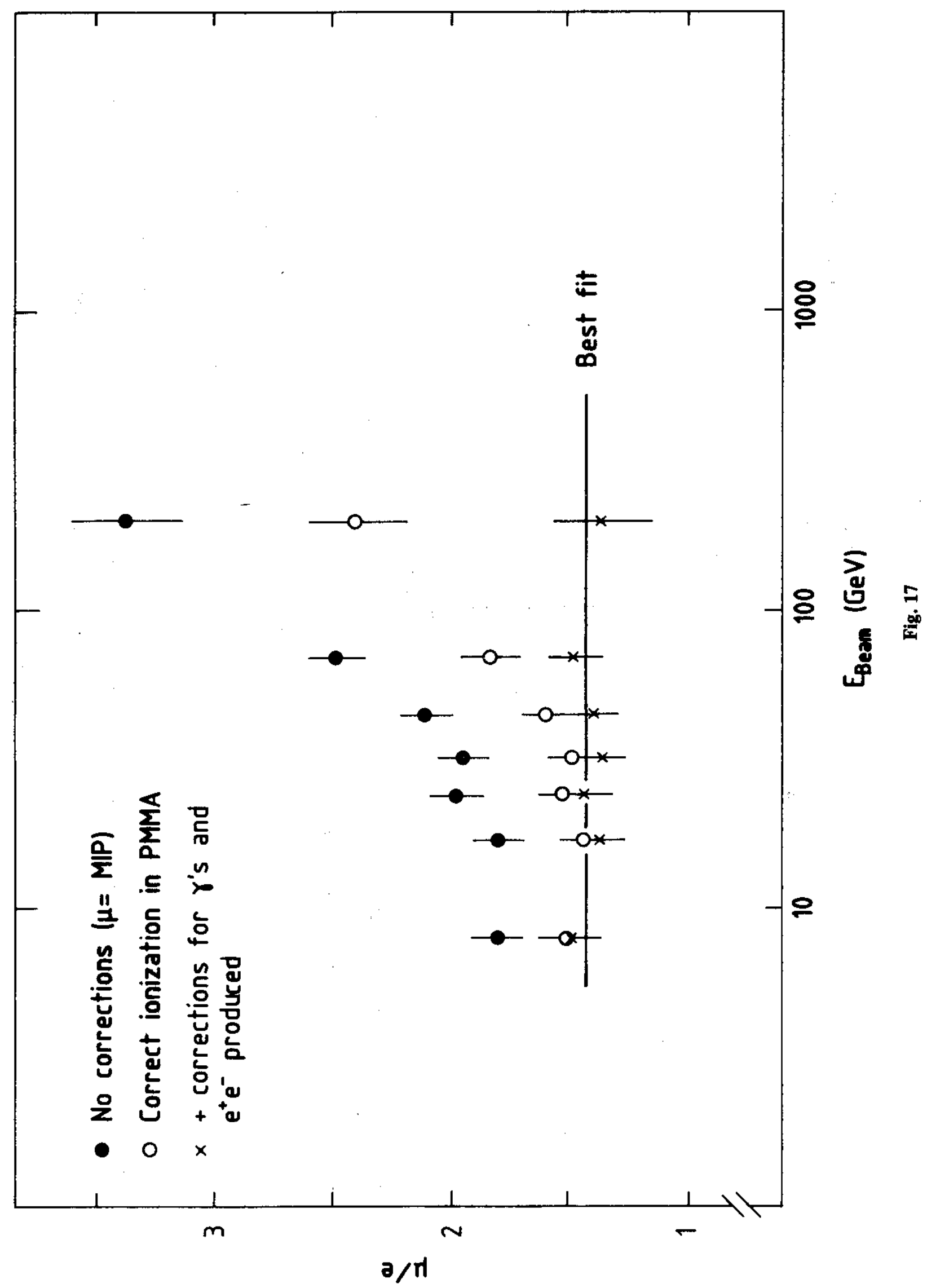



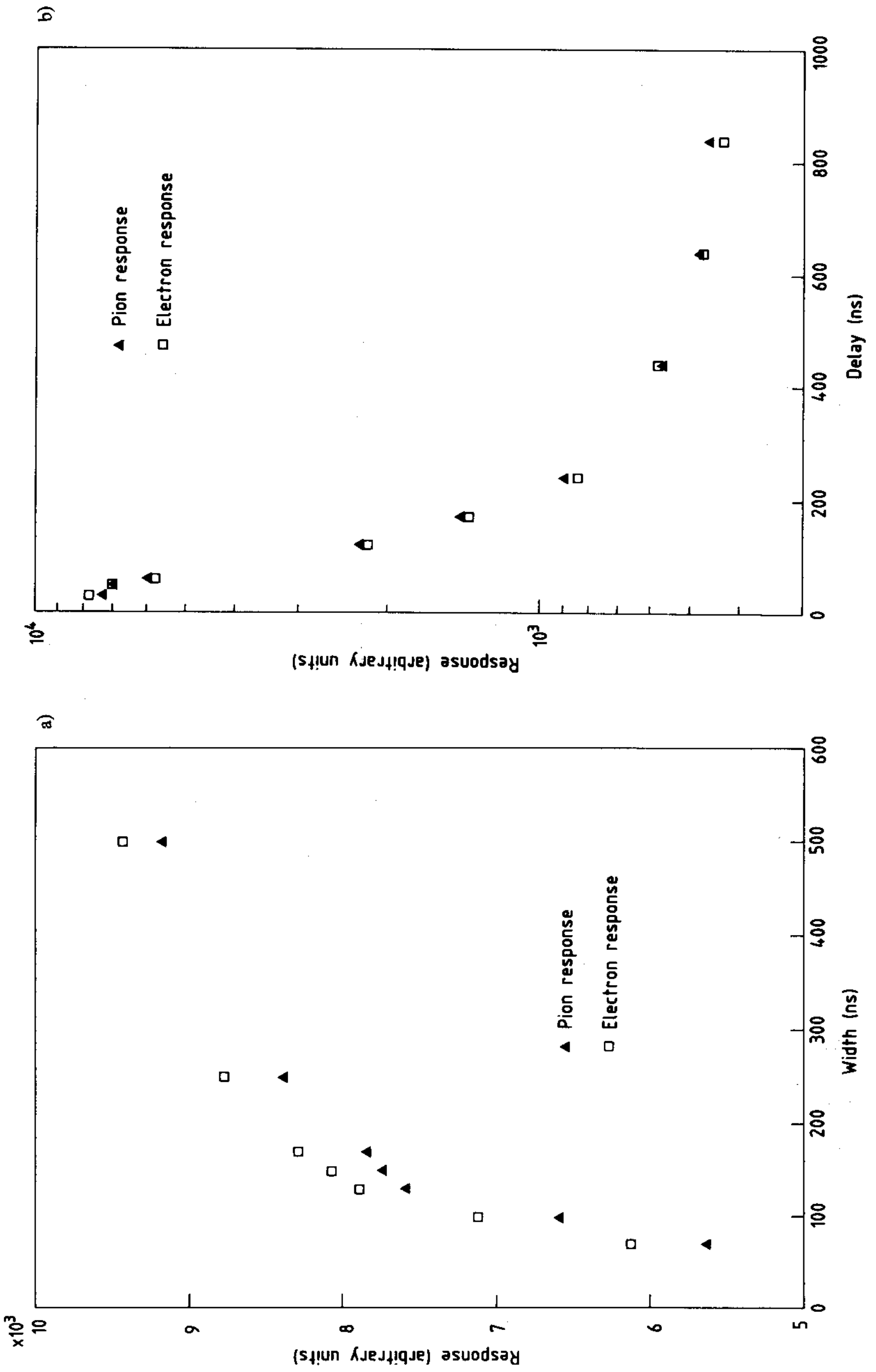


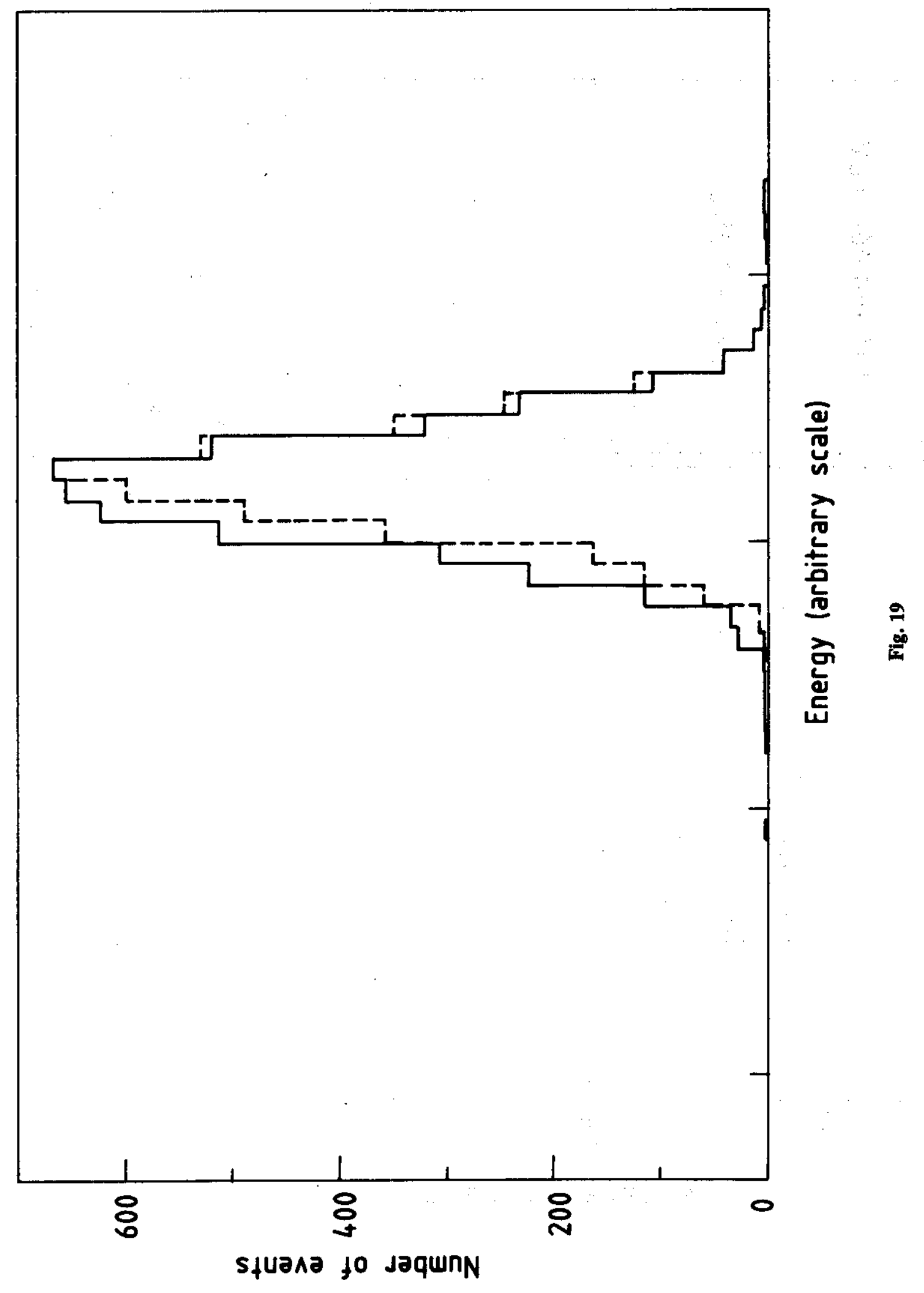




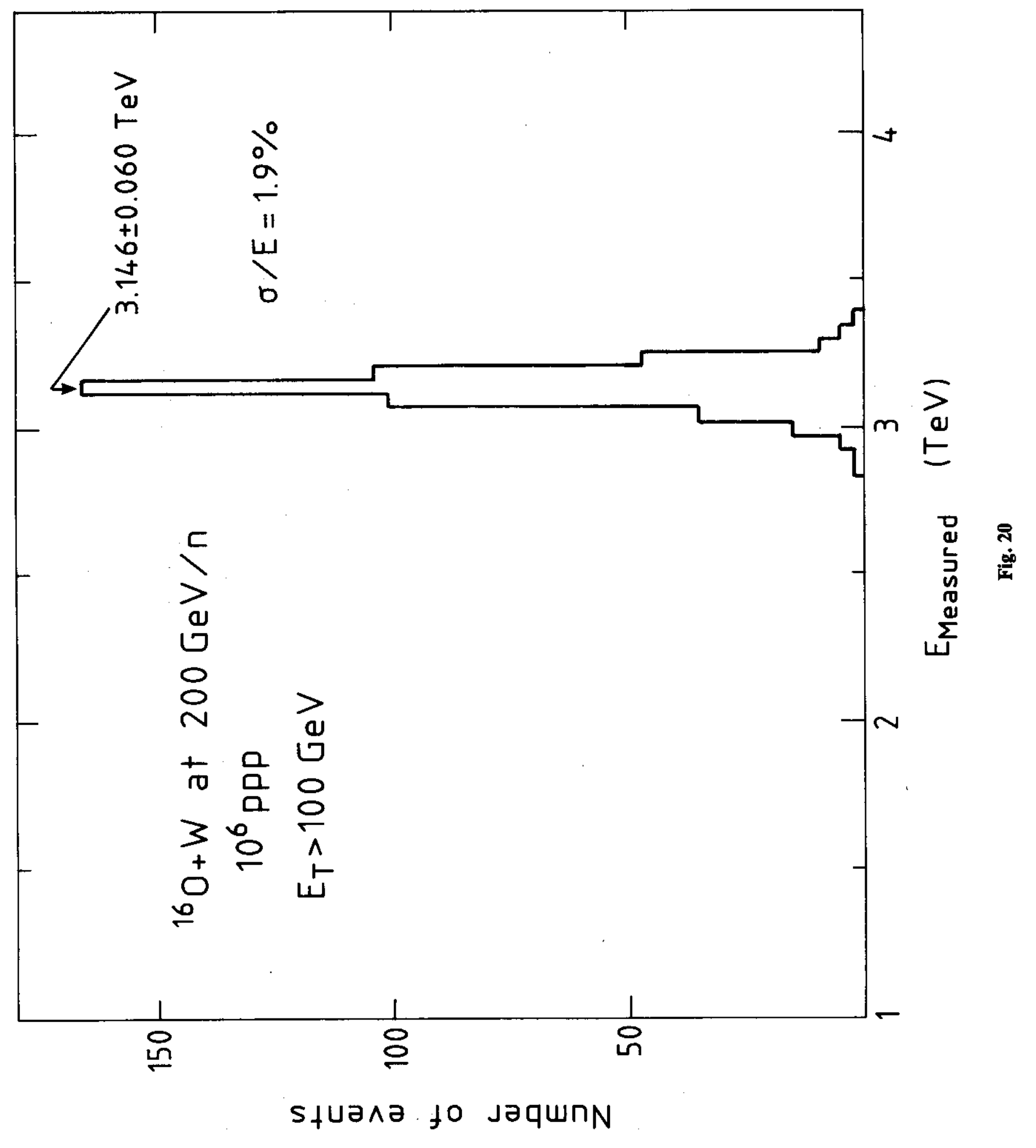




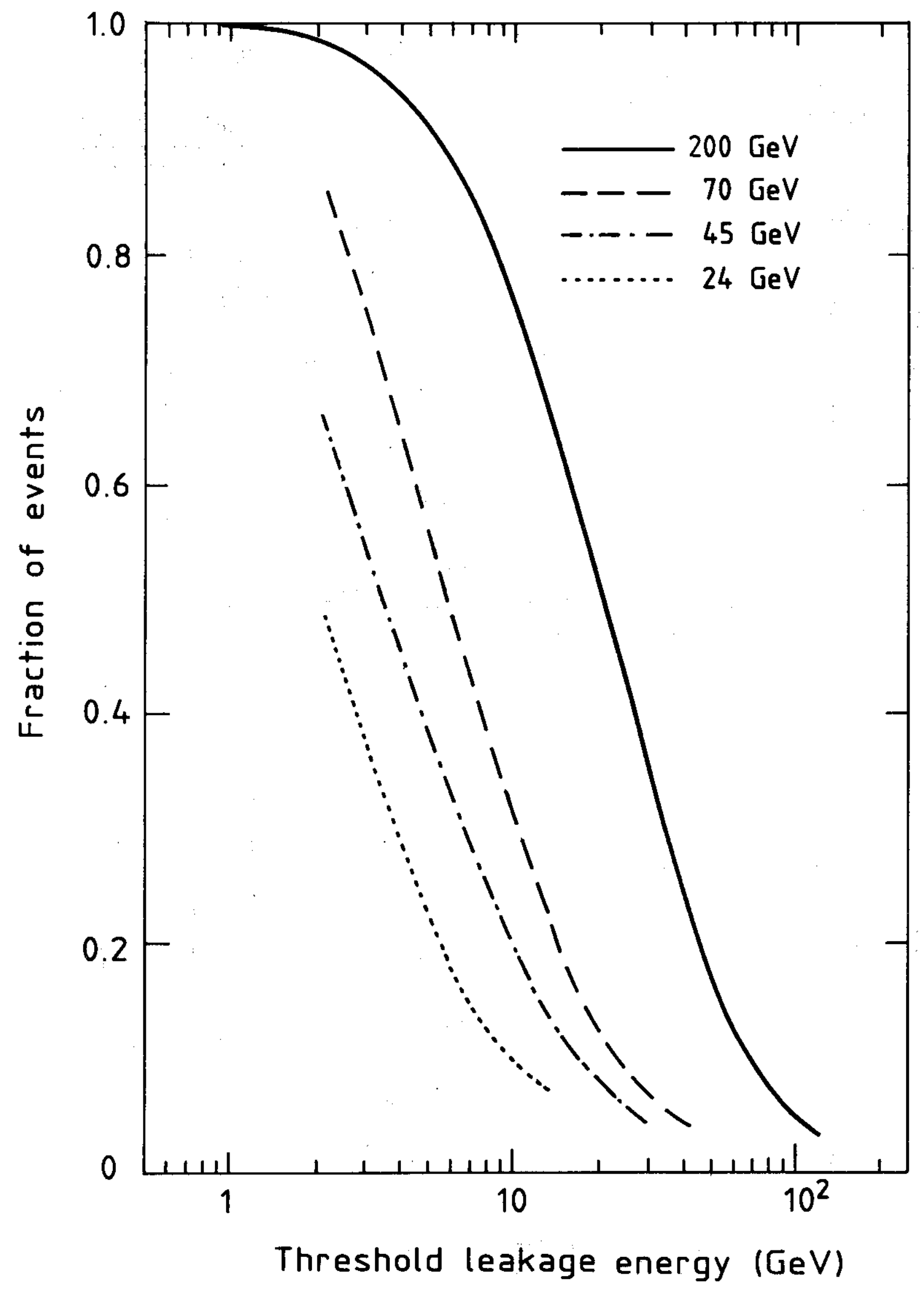

Fig. 21 


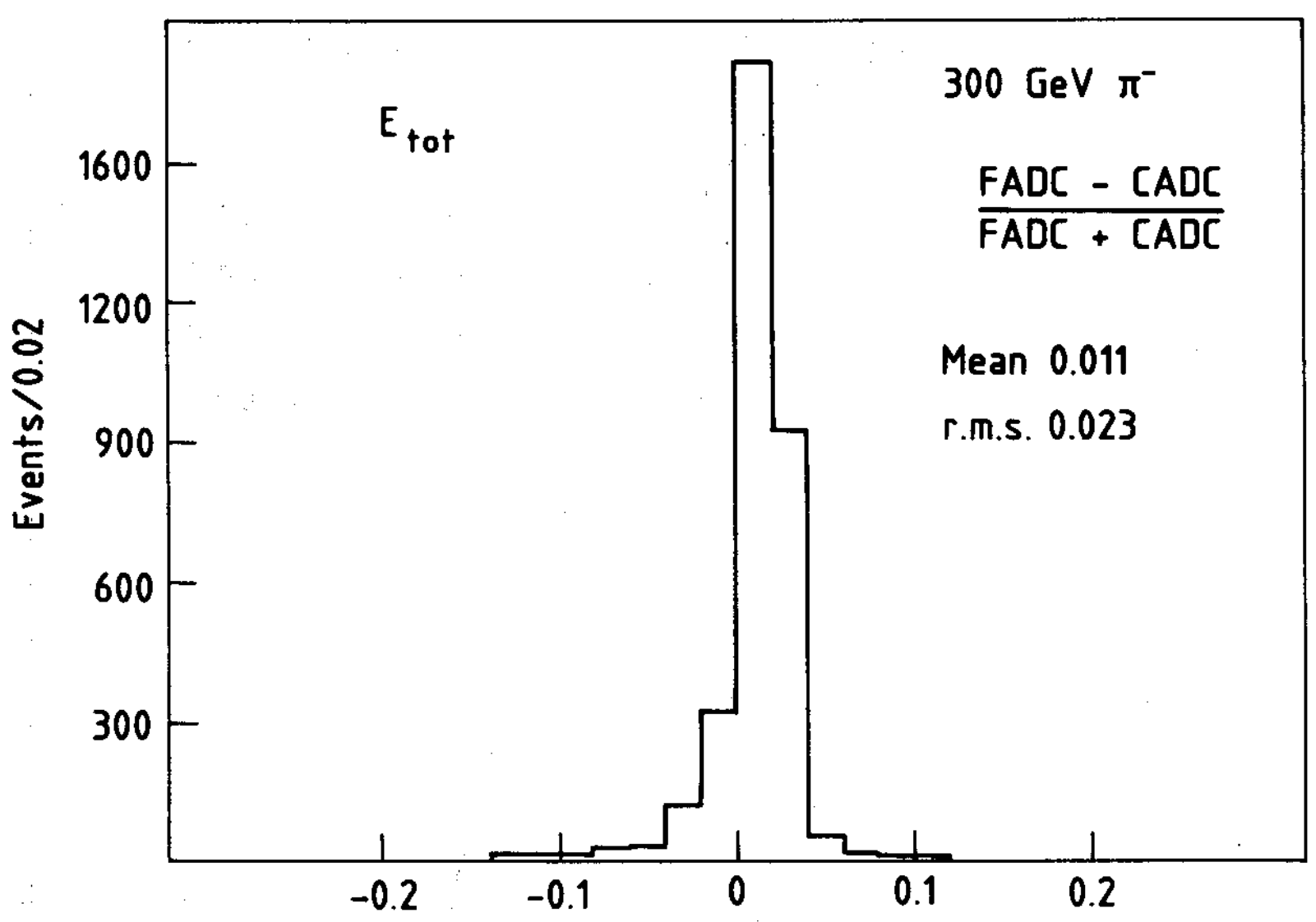

a)
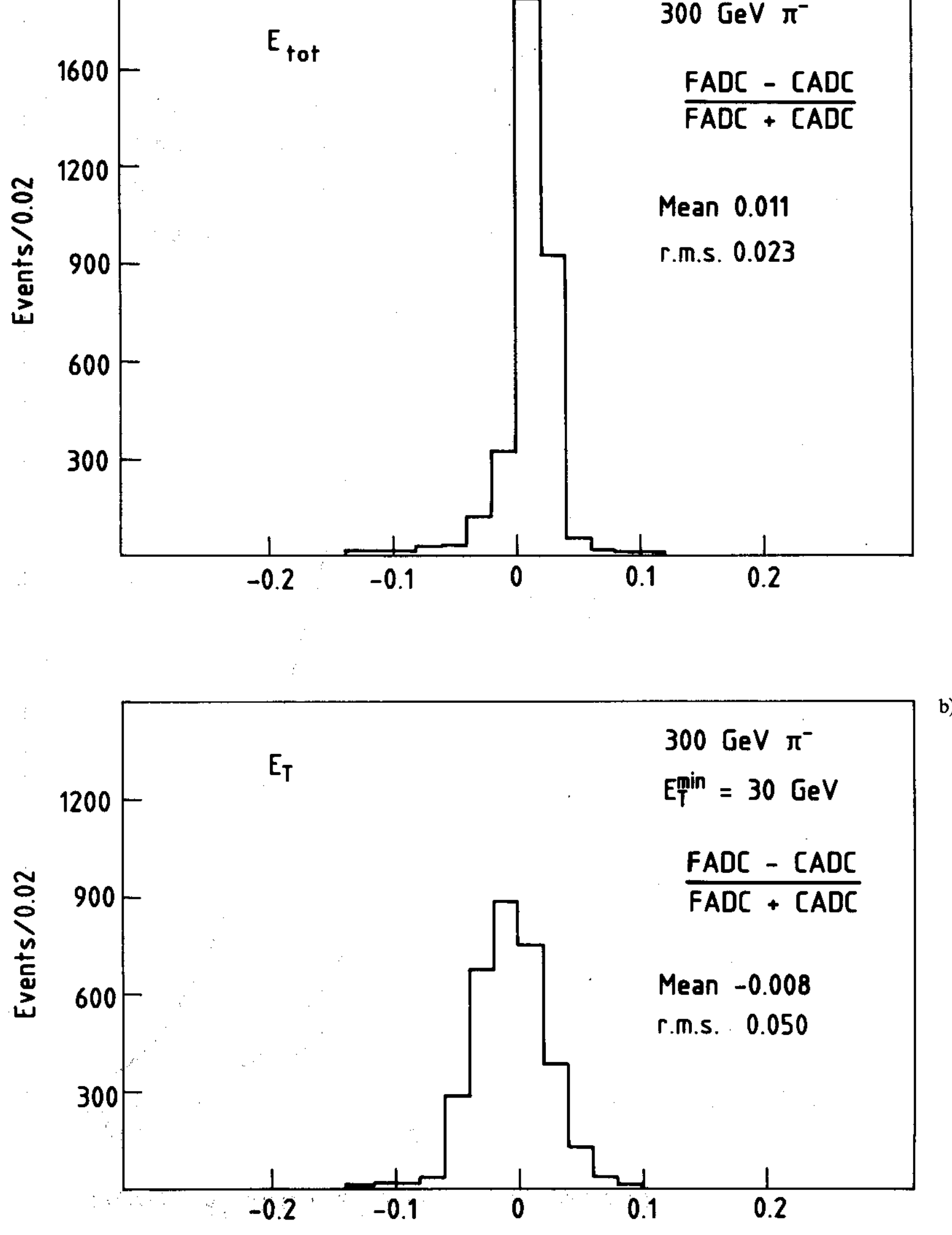

b)

Fig. 22 


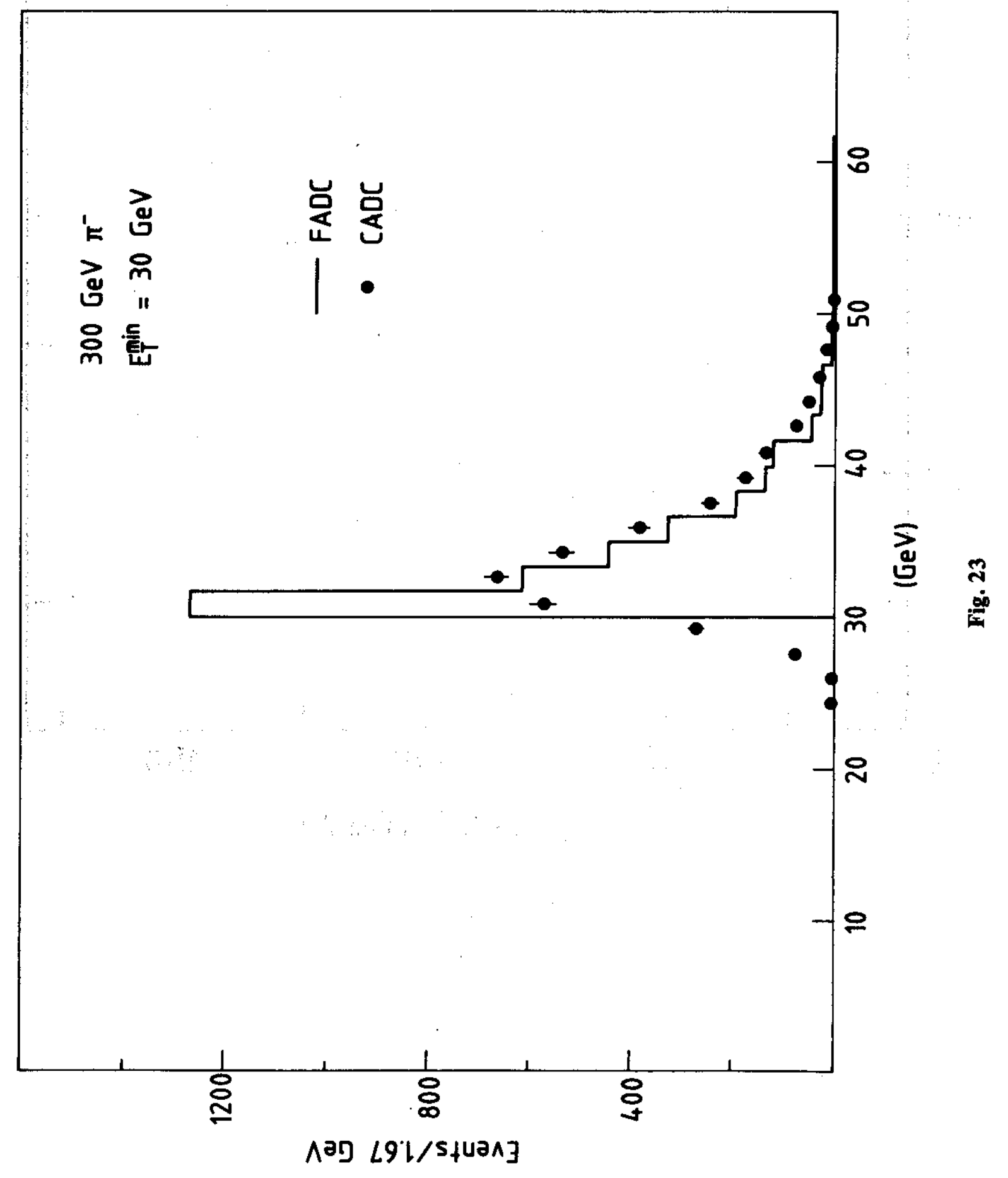




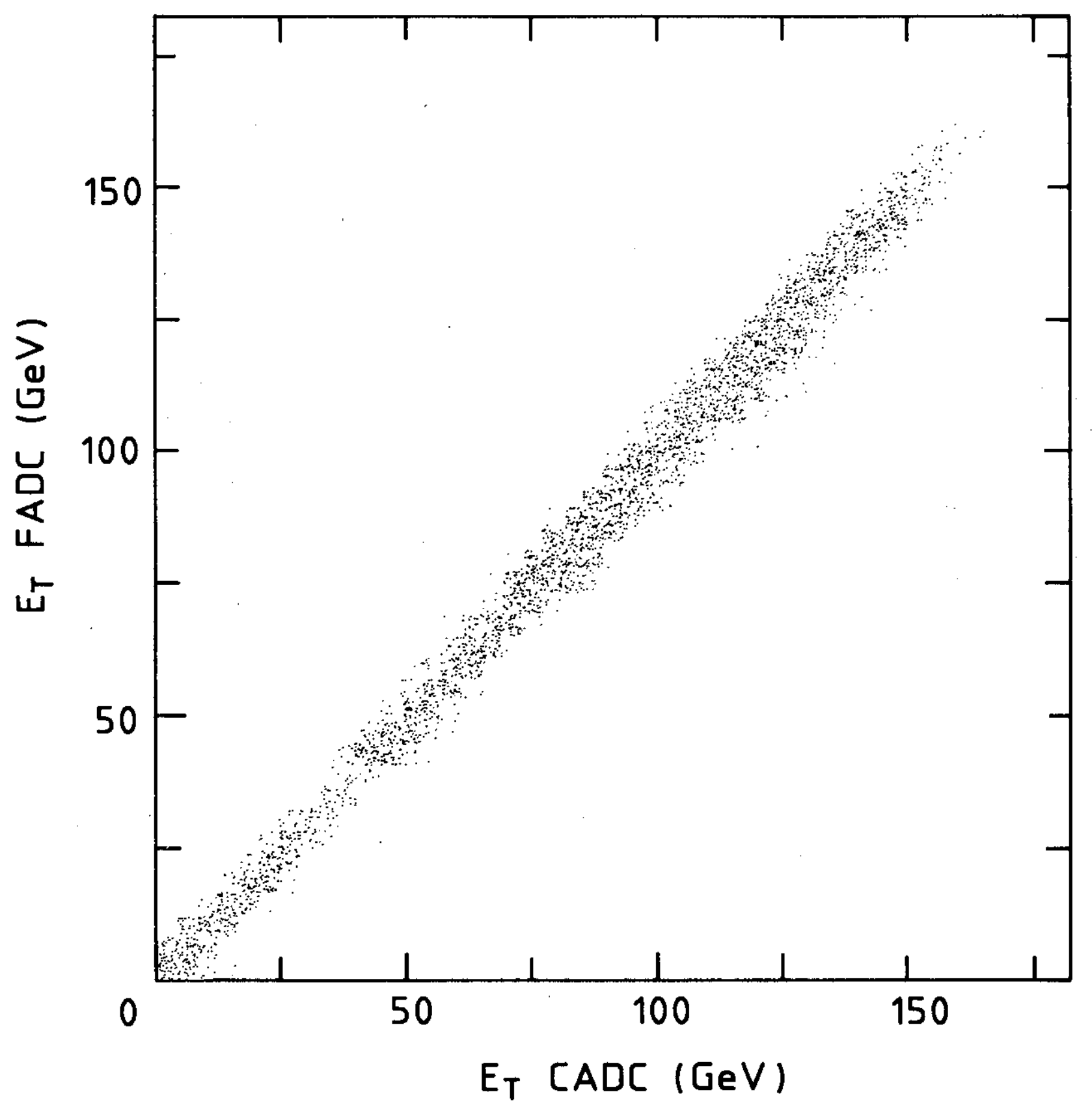

Fig. 24 a) 


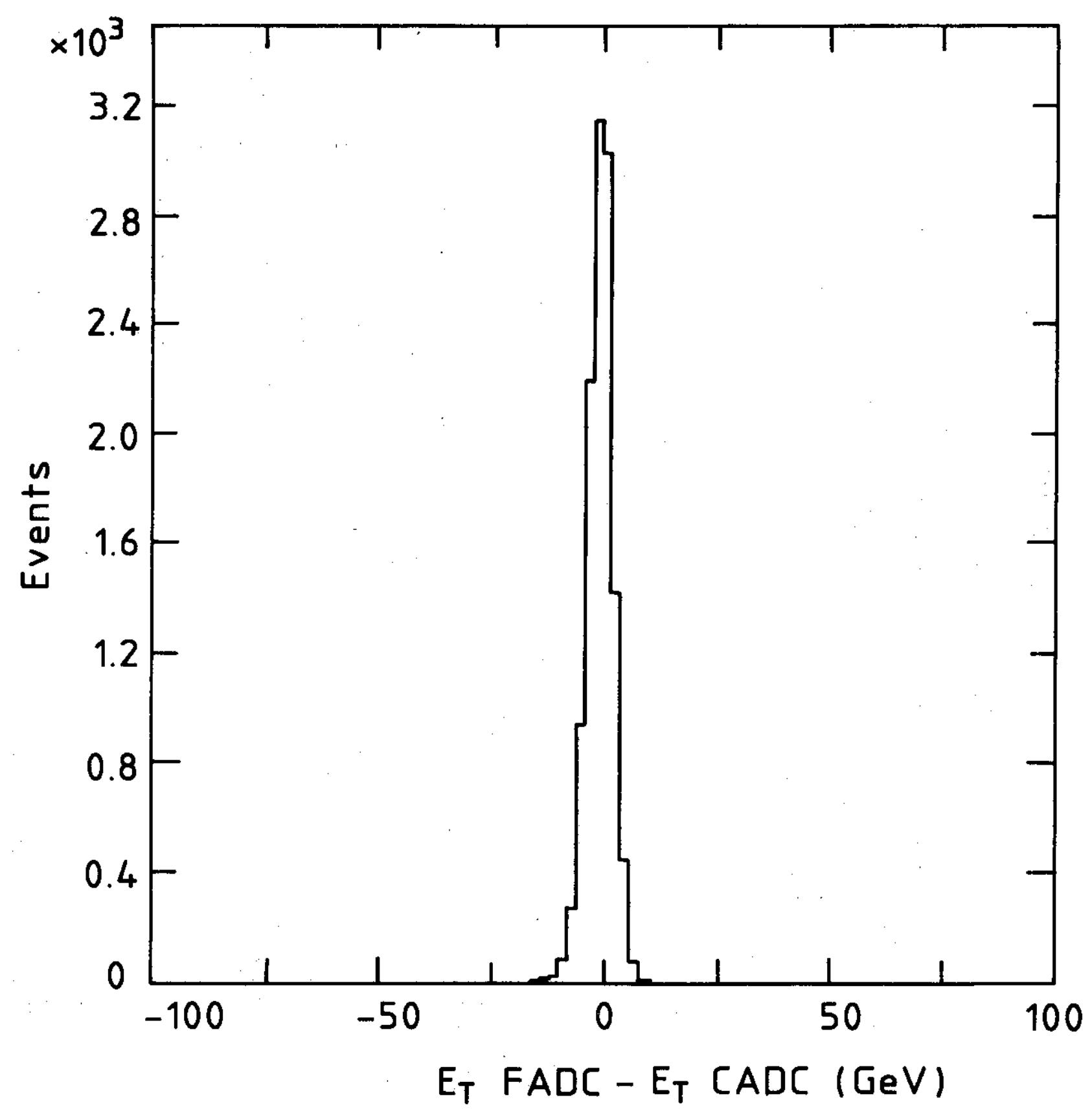

Fig. 24 b) 


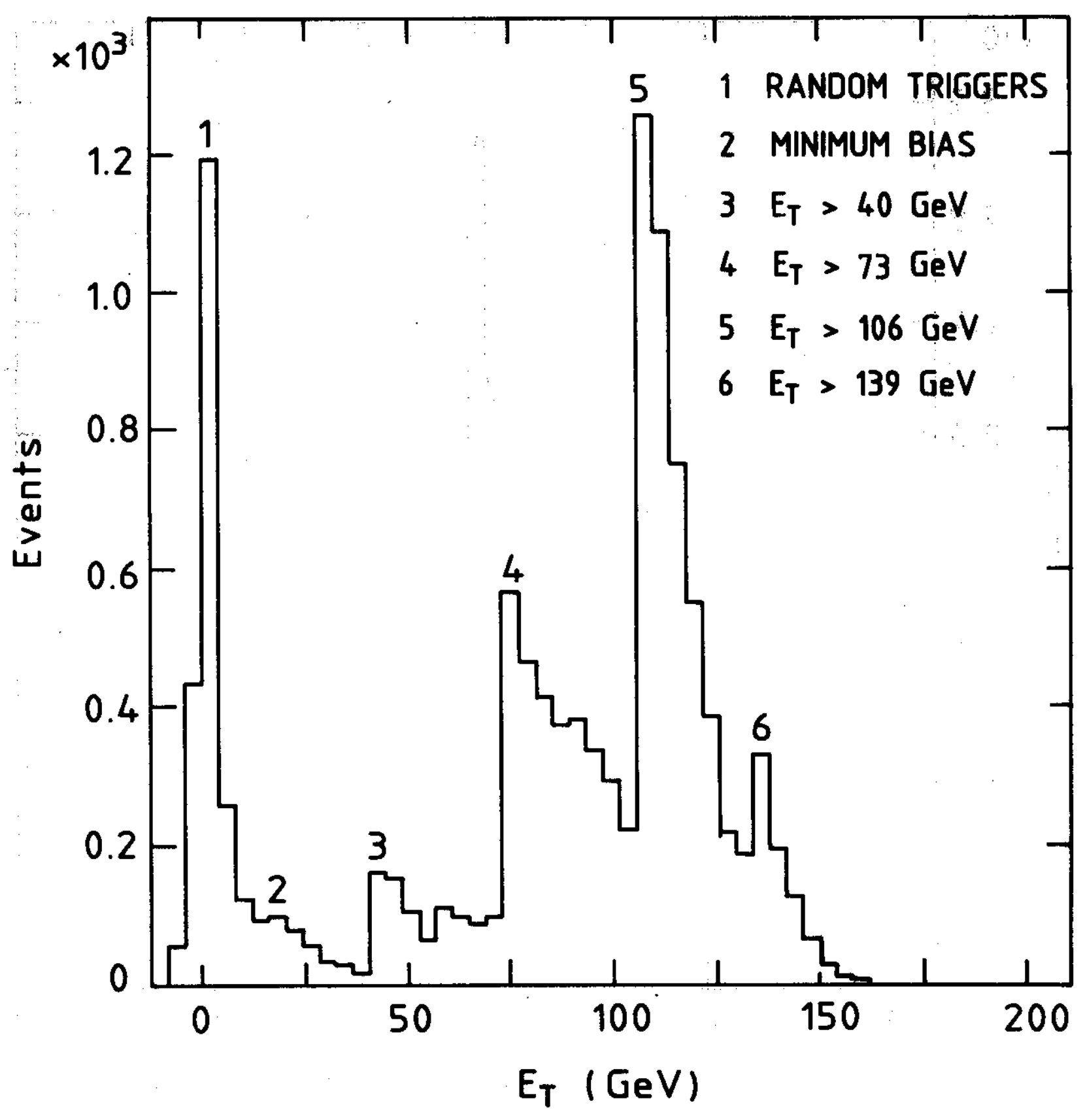

Fig. 25 